\title{
A stabilized nodal spectral solver for liquid chromatography models
}

\author{
Meyer, Kristian; Huusom, Jakob K.; Abildskov, Jens
}

Published in:

Computers \& Chemical Engineering

Link to article, DOI:

10.1016/j.compchemeng.2019.02.017

Publication date:

2019

Document Version

Peer reviewed version

Link back to DTU Orbit

Citation $(A P A)$ :

Meyer, K., Huusom, J. K., \& Abildskov, J. (2019). A stabilized nodal spectral solver for liquid chromatography models. Computers \& Chemical Engineering, 124, 172-183.

https://doi.org/10.1016/j.compchemeng.2019.02.017

\section{General rights}

Copyright and moral rights for the publications made accessible in the public portal are retained by the authors and/or other copyright owners and it is a condition of accessing publications that users recognise and abide by the legal requirements associated with these rights.

- Users may download and print one copy of any publication from the public portal for the purpose of private study or research.

- You may not further distribute the material or use it for any profit-making activity or commercial gain

- You may freely distribute the URL identifying the publication in the public portal

If you believe that this document breaches copyright please contact us providing details, and we will remove access to the work immediately and investigate your claim 


\section{Accepted Manuscript}

A stabilized nodal spectral solver for liquid chromatography models

Kristian Meyer, Jakob K. Huusom, Jens Abildskov

PII: S0098-1354(18)30947-5

DOI: https://doi.org/10.1016/j.compchemeng.2019.02.017

Reference: CACE 6355

To appear in:

Computers and Chemical Engineering

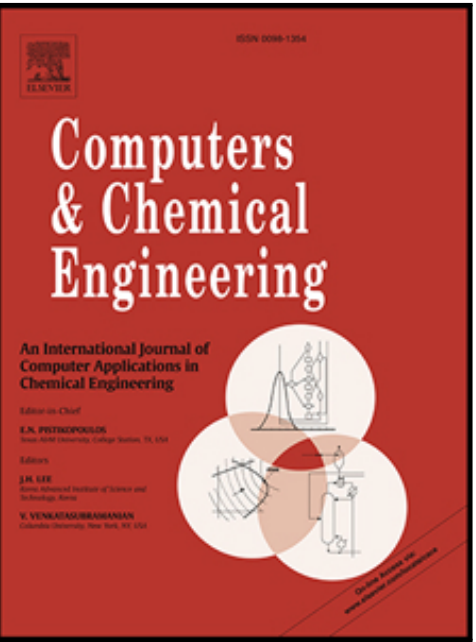

Received date: $\quad 7$ September 2018

Revised date: $\quad 1$ February 2019

Accepted date: $\quad 23$ February 2019

Please cite this article as: Kristian Meyer, Jakob K. Huusom, Jens Abildskov, A stabilized nodal spectral solver for liquid chromatography models, Computers and Chemical Engineering (2019), doi: https://doi.org/10.1016/j.compchemeng.2019.02.017

This is a PDF file of an unedited manuscript that has been accepted for publication. As a service to our customers we are providing this early version of the manuscript. The manuscript will undergo copyediting, typesetting, and review of the resulting proof before it is published in its final form. Please note that during the production process errors may be discovered which could affect the content, and all legal disclaimers that apply to the journal pertain. 


\section{Highlights}

- Numerical methods for solution of theequilibriumdispersive model of liquid chromatography are compared.

- Astabilized spectralmethod is applied and benchmarked against state-of-the-art finite element methods.

- The spectral method is demonstrated to be suitable for simulation of convection-dominated liquid chromatographic processes.

- Arbitrary high-order methods are shown to outperform the traditionally applied second-order methods in terms of computational cost and discrete problem size.

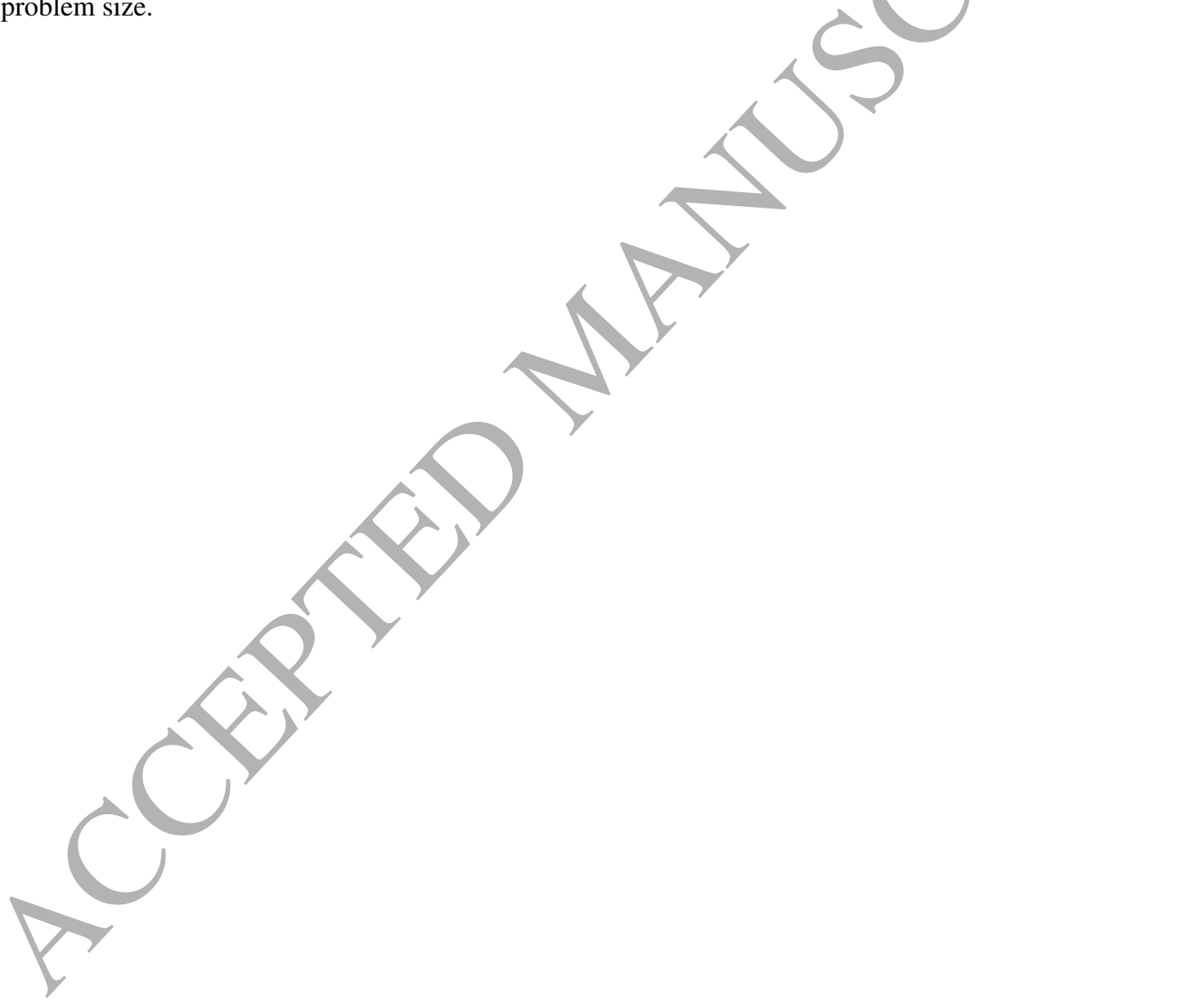




\title{
A stabilized nodal spectral solver for liquid chromatography models
}

\author{
Kristian Meyer $^{\mathrm{a}}$, Jakob K. Huusom ${ }^{\mathrm{a}}$, Jens Abildskov ${ }^{\mathrm{a}}$ \\ ${ }^{a}$ Process and Systems Engineering Center (PROSYS), Department of Chemical and Biochemical Engineering, Technical University of Denmark, \\ Building 229, 2800, Kgs. Lyngby, Denmark
}

\begin{abstract}
Numerical methods for simulation of linear and non-linear liquid chromatography processes are compared in terms of computational cost and quality of the solution using as few as possible degrees of freedom (discrete problem size). We apply, for the first time, the p-type (order enrichment) continuous Galerkin spectral method (p-CG) for spatial discretization with exponential decay of spatial errors (spectral convergence) for smooth problems. To stabilize the method for marginally resolved or very steep shock-waves typically encountered, we use a spectral filtering approach. This effectively removes aliasing driven instabilities while retaining the high-order accuracy of the numerical method. We benchmark the stabilized p-CG method against two state-of-the-art finite element methods, namely the secondorder accurate h-type (element refinement) discontinuous Galerkin method using a total-variation-diminishing limiter (h-DG-TVD) and the arbitrary-order hp-type discontinuous Galerkin spectral element method (hp-DG). Not surprisingly, the three methods provide predictions of column elution profiles that converge to the same values. However, the computational costs differ considerably. Although second-order accurate shock-capturing methods are often adopted as the default implementation, the stable arbitrary high-order methods considered in this paper are found to significantly outperform these in terms of both computational cost and discrete problem size. The p-CG method performs as well as the hp-DG method in terms of computational cost, but it has superior convergence properties, even in the presence of strong shock-waves. Further, its implementation is much simpler.
\end{abstract}

Keywords: High-order; Nodal continuous Galerkin Spectral method; Nodal discontinuous Galerkin Spectral element method; Liquid chromatography; Linear and nonlinear isotherm; Equilibrium-dispersive model;

\section{Introduction}

Liquid chromatography is a central unit operation in the fine chemicals, pharmaceutical and biotechnological industries for downstream processing. The concept is successfully used in difficult separation processes, such as separation of enantiomers or isolation of active pharmaceuticals from fermentation broth.

In liquid batch chromatography, a mobile phase percolates through a bed packed with stationary and porous particles. It carries/components from a feed mixture injected at the inlet of the column. The involved components interact differently with the stationary phase leading to characteristic retention behaviour. Conceptually, a complete separation is achieved for a sufficiently long column. The purified components are collected periodically at the column outlet.

A current trend in the fine chemicals, pharmaceutical and biotechnological industries is the digitalization of manufacturing processes to switch from a recipe-driven to a data-driven operational approach. This has great scope, when considering rapid cost-effective process development for speed-to-market and facilitation of nearoptimal operation using on-line monitoring strategies to control production costs. Digitalization of manufacturing processes has been successfully promoted over decades in other industries, such as the chemical and petrochemical industries. Provided that the necessary measurements are possible, the fine chemicals, pharmaceutical and biotechnological industries can reap similar benefits from digitalization.

The use of models of mechanistic nature for process prediction is key to successfully implement digital technology. A number of models have been successfully applied to model chromatographic process, such as the general rate model, various transport models, the equilibrium dispersive model and the ideal model (Guiochon et al. (2006)). These models include a description of both the fluid flow through the column and the column adsorption phenomena. The model complexity depends on how many band-broadening effects are 
taken into account, ranging from the ideal model where no band-broadening effects are included to the general rate model where diffusion, pore diffusion and mass transfer resistances are accounted for. The resulting models are described by systems of partial-differential equations (PDEs) or partial-differential-algebraic equations (PDAEs), depending on the model assumptions applied. Closed-form solutions are available only in special cases. Therefore, we have to rely on numerical techniques to obtain approximate solutions. When using mechanistic models for method development, the use of computer optimization is essential. Optimization algorithms require a large number of simulations, often thousands. Therefore, efficient simulation software is key to the successful implementation of digital chromatographic manufacturing.

\subsection{State-of-the-art simulation methods for liquid chromatography models}

Currently, (low-order) shock-capturing methods are widely adopted as the default implementation to approximate chromatographic models, ranging from finite volume flux-limiting methods (Medi and Amanullah (2011); Javeed et al. (2011b)), weighted essentially non-oscillatory (WENO) methods (von Lieres and Andersson (2010); Brown et al. (2018); Donat et al. (2018)), particle transport methods (Shipilova et al. (2008)), conservation element/solution element methods (Lim et al. (2004)) and Runge-Kutta total variation diminishing (TVD) discontinuous Galerkin (DG) finite element methods (Javeed et al. (2011a)). These methods are designed with a fixed order of accuracy, often second order (low-order). However, they achieve stable and non-oscillatory solutions, even when discontinuities are present in the solution profile. In chromatographic theory, only the ideal model predicts discontinuities in the concentration profiles. Here, a numerical scheme with convergence rate higher than first order $O(h)$ will produce persistent and artificial oscillations, known as the Gibbs phenomenon (Gottlieb and Shu (1997); Gottlieb and Hesthaven (2001)). To resolve this, a reconstruction or limiting procedure is required (a shock-capturing scheme) to obtain more than first-order accuracy. A strictly non-oscillatory scheme achieves up to second order (low-order) accuracy $O\left(h^{2}\right)$ whereas an essentially non-oscillatory scheme can be designed with higher-order accuracy (Harten et al. (1987)). However, when using a real chromatography column, discontinuities cannot form in the concentration profile and instead very steep band fronts/rears (shock waves) are observed (Ma and Guiochon (1992)). Thus, realistic applications are described by models that include one or more band-broadening effects. Furthermore, Danckwerts (1953) boundary conditions are considered valid (Guiochon et al. (2006)), such that discontinuous injection profiles (e.g. rectangular profiles) are dispersed immediately upon entering the column. Then, the Gibbs phenomenon is of less concern and arbitrary high-order methods can be used for cost-efficient simulations. Therefore, we (Meyer et al. (2018)) suggested to use a hp-type DG method, proposed by Hesthaven and Warburton $(2002,2008)$, to cost-efficiently predict realistic chromatographic systems. In short, the computational domain is partitioned into non-overlapping elements while the solution within an element is approximated by an $p$ th order polynomial. The analytical fluxes on element boundaries are undefined as a result of nonoverlapping elements and are instead replaced by numerical approximations. Convergence to a desired solution is obtained either by increasing the number of elements (h-type refinement) or by increasing the order of the polynomials (p-type refinement). For smooth solutions, p-type refinement is, in general, superior since it results in exponential decay of the error (spectral convergence). This enables the p-refinement strategy to reach the same level of accuracy with fewer degrees of freedom. A recent review of spectral element methods are given by e.g. Xu et al. (2018).

\subsection{Paper objectives}

The objective of this paper is to show that the classical p-type continuous Galerkin spectral method (p$\mathrm{CG}$ ) is robust and efficient for simulation of convectiondominated liquid chromatographic models. The pCG method has traditionally in chemical engineering software been considered for fully diffusive problems. Here, the method is efficient, since it achieves spectral convergence for smooth problems, and stable, due to the presence of sufficient physical dissipation. However, when applied to convection-dominated processes, aliasing errors can lead to numerical instabilities for marginally resolved or very steep shock waves. These aliasing-driven instabilities are effectively removed using e.g. spectral filtering techniques (Hesthaven and Kirby (2008)) while retaining high-order accuracy of the method. Thus, we propose, for the first time, to simulate linear and non-linear liquid chromatographic processes using a stabilized p-CG method.

To assess the performance of the stabilized p-CG method, we benchmark it against the hp-DG method and the second-order (low-order) accurate discontinuous Galerkin method using a total variation diminishing limiter (h-DG-TVD). Zhou et al. (2001) demonstrated, that the discontinuous Galerkin method us- 
ing a total-variation-bounded (TVB) limiter is usually more efficient compared to the finite volume method using WENO reconstruction. The TVD limiter will reduce the accuracy of the scheme to first order at smooth extremes, whereas the TVB limiter uses a tunable problem-dependent parameter to avoid this. In this work, we instead use the TVD limiter in combination with a shock-detector (a generalized TVD limiter) in order to avoid reducing the accuracy to first order at smooth extrema. Hence, we believe that our secondorder h-DG-TVD method is at least as efficient as the second-order finite volume method with WENO recontruction considered by other authors (von Lieres and Andersson (2010); Brown et al. (2018); Donat et al. (2018)).

We note that since we are applying a purely spectral method, we lose geometric flexibility. However, this is of less concern when considering the single domains used in the typical one-dimensional liquid chromatography models. In contrast to the hp-DG method, the p-CG can be applied without introducing any auxiliary variables, or rewriting the model problem into a larger system of equations saving computational cost. We are further motivated by the fact that the p-CG method is much simpler to implement and understand. In numerical experiments, we evaluate the performance of the three methods by their ability to translate a minimum amount of degrees-of-freedom (DoF) into a solution of sufficient quality (discrete problem size) and CPU, time. We prefer to represent the solution on a coarse grid since a smaller state-vector needs to be stored and integrated. This is especially computationally beneficial when considering large-scale dynamic optimization used e.g. to obtain discretized multi-level elution trajectories for high productivity and high yield batch chromatographic processes (Holmqvist and Magnusson (2016); Sellberg et al. (2017)) or for global downstream optimization (Huuk et al. (2014); Baumann et al. (2015); Pirrung et al. (2017)). It is also computationally advantageous when considering closed loop non-linear model predictive control (MPC) applications including state-estimation.

\subsection{Future solver capability considerations}

The hp-DG method supports complex geometries, whereas the purely spectral $\mathrm{p}-\mathrm{CG}$ method provides no such support. The typical liquid chromatography models considered in this paper predicts chromatographic behaviour for laboratory and industrial-scale applications with columns up to several litres of volume. These models are derived on simple one-dimensional grids where support for complex geometries are unnecessary. However, when considering miniaturized columns on micro-scale or full-scale industrial applications with volumes of up to thousands of litres, the simple onedimensional models cannot be used due to their simplifying assumptions. To simulate such processes, twodimensional models are required for accuracte predictions (Püttmann et al. (2014)). These two-dimensional models are solved on complex grids due to random packing of the stationary particles. Here, the hp-DG method can be naturally extended since it offers geometric flexibility, whereas the $\mathrm{p}-\mathrm{CG}$ can not.

Furthermore, the hp-DG method supports parallel implementations, while p-CG does not. While it is possible to increase the efficiency of the hp-DG method by parallel implementations, such implementations are beyond the scope of this article.

\subsection{Paper organization}

This paper is organized as follows: In section two we summarize the equilibrium-dispersive model together with a linear and a multicomponent Langmuir isotherm. In section three we derive the numerical methods used in section four to perform a comparative study in numerical experiments. Finally, concluding remarks are collected in section five.

\section{The equilibrium-dispersive model}

The equilibrium-dispersive model is based on the following assumptions:

1. Permanent equilibrium between bound (solid) and unbound (liquid) states at all positions in the column.

2. Isothermal chromatographic column.

3. The column is homogeneously packed with porous spherical particles of uniform size.

4. Compressibility of the mobile phase is negligible leading to a flat velocity profile.

5. No interaction between the solvent (mobile) and the solid (stationary) phase.

6. Only axial dispersion causes band broadening.

7. No radial concentration gradients in the column.

With these assumptions, the equilibrium-dispersive model involving $N_{c}$ components is given by (Guiochon et al. (2006))

$$
\frac{\partial \boldsymbol{c}}{\partial t}+F \frac{\partial \boldsymbol{q}}{\partial t}+u \frac{\partial \boldsymbol{c}}{\partial x}-D_{a} \frac{\partial^{2} \boldsymbol{c}}{\partial x^{2}}=0
$$


where $\boldsymbol{c}=\left(c_{1}, \cdots, c_{N_{c}}\right)^{T}$ and $\boldsymbol{q}=\left(q_{1}, \cdots, q_{N_{c}}\right)^{T}$ are unbound (liquid) and bound (stationary) phase concentrations, respectively, $F=\left(1-\epsilon_{c} /\right) \epsilon_{c}$ is the phase ratio, $\epsilon_{c}$ is the column porosity, $u$ is the interstitial velocity, $D_{a}$ is the apparent axial dispersion coefficient, $t$ is time, and $x$ is the space-coordinate.

In this paper we consider adsorption of multicomponent mixtures in which the adsorption equilibria between unbound $c_{i}$ and bound $q_{i}$ states are described by the multicomponent Langmuir isotherm (Guiochon et al. (2006))

$$
q_{i}=\frac{a_{i} c_{i}}{1+\sum_{j=1}^{N_{c}} b_{j} c_{j}}, \quad i=1, \ldots, N_{c}
$$

where $a_{i}$ is a Henry's constant and $b_{j}$ quantifies the nonlinearity of the isotherm. For dilute systems, Eq. (2) simplifies to a linear isotherm, given for the $i$ th component by

$$
q_{i}=a_{i} c_{i}
$$

It is assumed that the axial dispersion coefficient $D_{a}$ is related to the column efficiency as (Guiochon et al. (2006))

$$
D_{a}=\frac{L u}{2 N_{a}}
$$

with $N_{a}$ the number of apparent theoretical plates. The initial conditions for a pre-equilibrated column are given by

$$
c(0, x)=c_{\text {init }}(x)
$$

Finally, Danckwerts (1953) boundary conditions are applied. The inlet Robin condition is given by

$$
\left.u \boldsymbol{c}\right|_{x=0}-\left.D_{a} \frac{d \boldsymbol{c}}{d x}\right|_{x=0}=u \boldsymbol{c}_{i n j}(t)
$$

such that the injection profile $u c_{i n j}(t)$ is dispersed immediately upon entering the column. In this work, we consider rectangular injection profiles, e.g.

$$
u c_{i n j}= \begin{cases}u c_{f}, & 0 \leq t \leq t_{\text {inj }} \\ 0, & t>t_{\text {inj }}\end{cases}
$$

where $\boldsymbol{c}_{f}$ is the feed concentration. At the column outlet, a Neumann condition for finite column length $L$ is used

$$
\left.\frac{\partial \boldsymbol{c}}{\partial x}\right|_{x=L}=0
$$

\section{Numerical methods}

The equilibrium-dispersive model Eq. (1) is rewritten as follows

$$
\frac{\partial \boldsymbol{w}(\boldsymbol{c})}{\partial t}+u \frac{\partial \boldsymbol{c}}{\partial x}-D_{a} \frac{\partial^{2} \boldsymbol{c}}{\partial x^{2}}=0
$$

where $\boldsymbol{w}(\boldsymbol{c}):=\boldsymbol{c}+F \boldsymbol{q}(\boldsymbol{c})$ represents the total mass in the column. However, since an explicit relationship is missing for the function $\boldsymbol{c}(\boldsymbol{w})$ in the multicomponent case $\left(N_{c}>1\right)$, the chain rule may be applied to obtain,

$$
\left(1+F \frac{\partial \boldsymbol{q}(\boldsymbol{c})}{\partial \boldsymbol{c}}\right) \frac{\partial \boldsymbol{c}}{\partial t}+u \frac{\partial \boldsymbol{c}}{\partial x}-D_{a} \frac{\partial^{2} \boldsymbol{c}}{\partial x^{2}}=0
$$

Although this approach is simple, the formulation is non-conservative, e.g. mass can disappear even when using a conservative numerical method. Thus, we use instead the following implicit function for $\boldsymbol{c}(\boldsymbol{w})$ which ensures a conservative formulation (Donat et al. (2018)),

$$
c_{i}(\boldsymbol{w})=\frac{w_{i}}{1+F a_{i} \rho_{0}(\boldsymbol{w})}
$$

where $\rho_{0}(\boldsymbol{w})$ is the only positive root of the following rational function,

$$
R_{w}(y)=1-y+\sum_{i=1}^{N_{c}} \frac{y}{y+F a_{i}} b_{i} w_{i}
$$

and $1 \leq \rho_{0}(\boldsymbol{w}) \leq 1+\sum_{j} b_{j} w_{j}$. We approximate the only positive root of Eq. (12) using a non-linear solver based on Kelley's (2003) nsold algorithm, which is a Newton's method using Newton-Armijo iterations, adaptive Jacobian updates and parabolic line search via 3-point interpolation.

In the following subsections, we consider the approximation of Eq. (9) using a method-of-lines approach, e.g. the spatial and temporal discretizations are considered separately. The p-CG, hp-DG and the h-DG-TVD methods are applied for spatial discretization to obtain a semi-descrete set of ordinary differential equations (ODEs). The semi-discrete ODE system is integrated from appropriate initial conditions using a 5-stage, 4thorder low-storage explicit Runge Kutta (ERK) method (Carpenter and Kennedy (1994)). The solution to Eq. (9) contains the total mass $\boldsymbol{w}$ inside the column. Eq. (11) is applied to obtain concentrations in the mobile phase $\boldsymbol{c}$ based on the total mass $\boldsymbol{w}$.

\subsection{The continuous Galerkin spectral method}

\subsubsection{General formulation}

Follwing the p-CG discretization procedure (Kopriva (2009)), we multiply Eq. (9) by an arbitrary smooth test function $v$ and integrate over the computational domain $\Omega \in[0, L]$, to obtain a weak form

$$
\int_{\Omega} \frac{\partial \boldsymbol{w}(\boldsymbol{c})}{\partial t} v d x+\int_{\Omega} \frac{\partial \boldsymbol{f}(\boldsymbol{c}, \partial \boldsymbol{c} / \partial x)}{\partial x} v d x=0
$$


where we have defined the flux $\boldsymbol{f}(\boldsymbol{c}, \partial \boldsymbol{c} / \partial x):=u \boldsymbol{c}-$ $D_{a} \partial \boldsymbol{c} / \partial x$. Applying spatial integration by parts on Eq. (13) gives

$$
\int_{\Omega} \frac{\partial \boldsymbol{w}(\boldsymbol{c})}{\partial t} v d x-\int_{\Omega} \boldsymbol{f} \frac{\partial v}{\partial x} d x=\oint_{\partial \Omega} \hat{\boldsymbol{n}} v \boldsymbol{f} d x
$$

where $\partial \Omega$ represent the domain boundaries, and $\hat{\boldsymbol{n}}$ is the unit outward normal vector to the boundary.

\subsubsection{Nodal discretization}

The exact solution $\boldsymbol{c}$, is approximated by a numerical solution $\boldsymbol{c}_{h}$, which we assume is a finite sum of the form

$$
\begin{gathered}
\boldsymbol{c}(t, x) \approx \boldsymbol{c}_{h}(t, x)=\sum_{n=1}^{N_{p}} \hat{\boldsymbol{c}}_{h, n}(t) \phi_{n}(x) \\
=\sum_{n=1}^{N_{p}} \boldsymbol{c}_{h}\left(t, x_{n}\right) l_{n}(x)=\sum_{n=1}^{N_{p}} \boldsymbol{c}_{h, n}(t) l_{n}(x)
\end{gathered}
$$

where $\hat{\boldsymbol{c}}_{h, n}$ and $\boldsymbol{c}_{h, n}$ are $N_{p}$ unknown modal and nodal coefficients, respectively. $\phi_{n}$ are the chosen basis functions which we assume equal to our test functions (the Galerkin method) and $l_{n}$ are the associated Langrange interpolating polynomials with the property $l_{i}\left(z_{j}\right)=\delta_{i j}$ ( $\delta_{i j}$ is a Kronecker delta) at mesh nodes. The basis functions $\phi_{n}$ are chosen such that the numerical solution $\boldsymbol{c}_{h}$ belongs to the following approximation space,

$$
\mathbb{V}_{p}^{k}=\left\{v: v \in \mathbb{P}^{p}(\Omega)\right\}
$$

where $\mathbb{P}^{p}(\Omega)$ is a polynomial of degree at most $p=N_{p}$ 1 defined in $\Omega$. The following orthonormal polynomial basis functions are chosen,

$$
\phi_{n}(r)=\frac{P_{n-1}(r)}{\sqrt{\gamma_{n-1}}}=\tilde{P}_{n-1}(r), \quad r \in I=[-1,1]
$$

where $P_{n-1}(r)$ are the classical Legendre polynomials defined on the reference element $I$ and $\gamma_{n}=2 /(2 n+1)$ is the normalization. We define the vectors of nodal values on $I$ as

$$
\boldsymbol{c}_{h}^{I}=\left(\boldsymbol{c}_{h}\left(r_{1}\right), \ldots, \boldsymbol{c}_{h}\left(r_{N_{p}}\right)\right)^{T}
$$

the vectors of modal values on $I$ as

$$
\hat{\boldsymbol{c}}_{h}^{I}=\left(\hat{\boldsymbol{c}}_{h}\left(r_{1}\right), \ldots, \hat{\boldsymbol{c}}_{h}\left(r_{N_{p}}\right)\right)^{T}
$$

and the vectors of local Lagrange interpolating polynomials and basis functions on $I$ by

$$
\boldsymbol{l}=\left[l_{1}(r), \ldots, l_{N_{p}}(r)\right]^{T}, \quad \tilde{\boldsymbol{P}}=\left[\tilde{P}_{0}(r), \ldots, \tilde{P}_{N}(r)\right]
$$

Further, we introduce the mass, stiffness and lifting matrices defined on $I$ in Eqs. (21a)-(21c), respectively.

$$
\begin{aligned}
\mathcal{M}_{i j} & =\int_{-1}^{1} l_{i}(r) l_{j}(r) d r \\
\mathcal{S}_{i j} & =\int_{-1}^{1} l_{i}(r) \frac{d l_{j}}{d r}(r) d r \\
\mathcal{L} & =\mathcal{M}^{-1} \mathcal{E} \\
\mathcal{E}_{i 1} & =l_{i}(-1), \quad \mathcal{E}_{i 2}=l_{i}(1)
\end{aligned}
$$

Finally, we define the Vandermonde matrix with entries

$$
\mathcal{V}_{i j}=\tilde{P}_{j-1}\left(r_{i}\right)
$$

Here, we choose to represent the nodal set $r=$ $\left(r_{1}, \ldots, r_{N_{p}}\right)^{T}$ inside the reference element $I$ using the Legendre-Gauss-Lobatto quaddrature points to ensure a well-conditioned Vandermonde matrix. This allows us to use operations requiring the inverse of the Vandermonde matrix $Y^{-1}$. The Vandermonde matrix is used to transform between modal and nodal forms and to relate the basis functions (Hesthaven and Warburton (2002)), e.g.

$$
\boldsymbol{c}_{h}^{I}=\mathcal{V} \hat{\boldsymbol{c}}_{h}^{I}, \quad \hat{\boldsymbol{c}}_{h}^{I}=\mathcal{V}^{-1} \boldsymbol{c}_{h}^{I}, \quad \tilde{\boldsymbol{P}}=\mathcal{V}^{T} \boldsymbol{l}
$$

Eq. (23) establishes the following relationships to compute the mass- and stiffness matrices for a standard element without using high-order Gaussian quadrature rules for the element integrals, leaving the implementation quadrature-free:

$$
\mathcal{M}=\left(\mathcal{V} \mathcal{V}^{T}\right)^{-1}, \quad \mathcal{S}=\mathcal{M} \mathcal{D}_{r}
$$

Here, we have defined the differentiation matrix with entries

$$
\mathcal{D}_{r}=\left.\frac{d l_{j}}{d r}\right|_{r_{i}}
$$

The differentiation matrix is used to transform point values to derivatives, e.g. $d \boldsymbol{c}_{h}^{I} / d r=\mathcal{D}_{r} \boldsymbol{c}_{h}^{I}$. It is computed by

$$
\mathcal{D}_{r}=\mathcal{V}_{r} \mathcal{V}^{-1}
$$

where $\mathcal{V}_{r}$ is a matrix with entries given by the derivative of the basis,

$$
\mathcal{V}_{r,(i, j)}=\left.\frac{d \tilde{P}_{j}}{d r}\right|_{r_{i}}
$$

To rewrite Eq. (14) in compact form the continuous variables are replaced by the finite sum given by Eq. (15) and the above defined (constant) local element matrices are used. After rearrangement, we arrive at

$$
\frac{d \boldsymbol{W}_{h}}{d t}=\frac{2}{L} \hat{\mathcal{M}}^{-1} \hat{\mathcal{S}}^{T} \boldsymbol{F}_{h}+\frac{2}{L} \hat{\mathcal{L}}\left[\begin{array}{c}
-\left.\boldsymbol{f}_{h}\right|_{x=0} \\
\left.\boldsymbol{f}_{h}\right|_{x=L}
\end{array}\right]
$$


where

$$
\begin{aligned}
& \boldsymbol{W}_{h}=\left(\boldsymbol{w}_{h}\left(x_{1}\right), \ldots, \boldsymbol{w}_{h}\left(x_{N_{p}}\right)\right)^{T} \\
& \boldsymbol{F}_{h}=\left(\boldsymbol{f}_{h}\left(x_{1}\right), \ldots, \boldsymbol{f}_{h}\left(x_{N_{p}}\right)\right)^{T}
\end{aligned}
$$

are nodal values defined on the domain $\Omega$ and $\hat{\mathcal{M}}=$ $\operatorname{diag}(\mathcal{M}), \hat{\mathcal{S}}=\operatorname{diag}(\mathcal{S})$ and $\hat{\mathcal{L}}=\operatorname{diag}(\mathcal{L})$ are block diagonal matrices with $N_{c}$ blocks. We note that since $\hat{\mathcal{M}}$ is block-diagonal, it is easily invertible such that the semidiscrete ODE system is explicit. The flux $\boldsymbol{f}_{h}$ is computed by

$$
\boldsymbol{f}_{h}=u \boldsymbol{c}_{h}-D_{a} \frac{2}{L} \mathcal{D}_{r} \boldsymbol{c}_{h}
$$

where we have used the differentiation matrix $\mathcal{D}_{r}$ to evaluate the nodal spatial derivatives of $\boldsymbol{c}_{h}$. In Eqs. (28) and (31) the factor $2 / L$ is the inverse Jacobian of the affine transformation,

$$
x(r)=\frac{(1+r) L}{2}
$$

used to map the reference element $I$ to the physical element $\Omega$. Eq. (28) represents a semi-discrete system of $N_{p}$ ODEs for $N_{p}$ unknowns.

\subsubsection{Stabilization}

To stabilize the scheme, we apply a modal filter(Hesthaven and Kirby (2008); Hesthaven and Warburton (2008)) to the numerical approximation at regular intervals. We construct the filter matrix as

$$
\mathcal{F}=\mathcal{V} \Lambda \mathcal{V}^{-1}
$$

with the diagonal matrix $\Lambda$

$$
\Lambda=\delta_{i j} \sigma(i-1), \quad i, j=1, \ldots, N_{p}
$$

The modal filter coefficients $0 \leq \sigma_{i} \leq 1$ are given by

$$
\sigma_{i}= \begin{cases}1 & 0 \leq i \leq i_{c} \\ \exp \left(-\alpha\left(\left(i-i_{c}\right) /\left(p-i_{c}\right)\right)^{s}\right) & i_{c} \leq i \leq p\end{cases}
$$

where $\alpha$ is a parameter that measures the modification of the maximum mode, i.e. $\sigma(p)=\exp (-\alpha)$. We define $\alpha=-\log \left(\epsilon_{m}\right)$ with $\epsilon_{m}$ the machine accuracy. The parameter $s$ is the (even) order of the filter, and determines the strength of it (higher $s$ means weaker filtering). $i_{c}$ represents a cut-off index, below which the low modes are left untouched. The dissipation required for stabilization is then introduced by a cheap matrix-vector operation:

$$
\overline{\boldsymbol{W}}_{h}=\mathcal{F} \boldsymbol{W}_{h}
$$

\subsubsection{Boundary conditions}

Danckwerts (1953) boundary conditions Eqs. (6) and (8) are imposed by replacing the inlet flux with,

$$
\left.\boldsymbol{f}_{h}\right|_{x=0}=u c_{i n j}(t)
$$

such that the combined convective and diffusive flux $\boldsymbol{f}_{h}$ just inside the column equals the inlet flux. The outlet Neumann condition is imposed by replacing the outlet flux as

$$
\left.\boldsymbol{f}_{h}\right|_{x=L}=\left.u c_{h}\right|_{x=L}
$$

such that the diffusive part of the flux is zero on the outlet.

\subsection{The $\mathrm{hp}$ discontinuous Galerkin method}

\subsubsection{General formulation}

To apply the hp-DG method on second order differential operators, we follow the approach of Bassi and Rebay (1997) and rewrite Eq. (9) as a first order system,

$$
\begin{aligned}
& \frac{\partial \boldsymbol{w}(\boldsymbol{c})}{\partial t}+\frac{\partial \boldsymbol{h}(\boldsymbol{c}, \boldsymbol{g})}{\partial x}=0 \\
& \boldsymbol{g}(\boldsymbol{c})-\sqrt{D_{a}} \frac{\partial \boldsymbol{c}}{\partial x}=0
\end{aligned}
$$

where we have defined the flux $\boldsymbol{h}(\boldsymbol{c}, \boldsymbol{g}):=u \boldsymbol{c}-\sqrt{D_{a}} \boldsymbol{g}$ and $\boldsymbol{g}$ are auxiliary gradient variables. Before applying the DG method to Eq. (39), we divide the computational domain $\Omega$ into $K$ non-overlapping elements, such that $\Omega=\bigcup_{k=1}^{K} \Omega^{k}$ with $\Omega^{k}=x_{R}^{k}-x_{L}^{k}=\Delta x^{k}$.

Following the DG procedure, we multiply Eq. (39) by a smooth test function $v$ and integrate over the element $\Omega^{k}$. After applying spatial integration by parts, we obtain the local weak form

$$
\begin{aligned}
& \int_{\Omega^{k}} \frac{\partial \boldsymbol{w}}{\partial t} v d x-\int_{\Omega^{k}} \boldsymbol{h} \frac{\partial v}{\partial x} d x=-\oint_{\partial \Omega^{k}} v \hat{\boldsymbol{n}} \boldsymbol{h}^{*} d x \\
& \int_{\Omega^{k}} \boldsymbol{g} v d x+\sqrt{D_{a}} \int_{\Omega^{k}} \boldsymbol{c} \frac{\partial v}{\partial x} d x=\sqrt{D_{a}} \oint_{\partial \Omega^{k}} v \hat{\boldsymbol{n}} \boldsymbol{c}^{*} d x
\end{aligned}
$$

Since we have an approximation space that is discontinuous across element interfaces $\partial \Omega^{k}$, we replace the nondefined analytical fluxes $\hat{\boldsymbol{n}} \boldsymbol{h}$ and $\hat{\boldsymbol{n}} \sqrt{D_{a}} \boldsymbol{c}$ by uniquely defined numerical fluxes $\hat{\boldsymbol{n}} \boldsymbol{h}^{*}$ and $\hat{\boldsymbol{n}} \sqrt{D} \boldsymbol{c}^{*}$, respectively. Suitable numerical fluxes are chosen in section 3.2.3.

\subsubsection{Nodal discretization}

On the $k$ th element, we approximate the exact solution $\boldsymbol{Q}=[\boldsymbol{c}, \boldsymbol{g}]^{T}$ by a numerical solution $\boldsymbol{Q}_{h}^{k}=\left[\boldsymbol{c}_{h}^{k}, \boldsymbol{g}_{h}^{k}\right]^{T}$, 
which we assume is a finite sum of equivalent form as in Eq. (15), e.g.

$$
\begin{aligned}
& \boldsymbol{Q}(t, x) \approx \boldsymbol{Q}_{h}^{k}(t, x)=\sum_{n=1}^{N_{p}} \hat{\boldsymbol{Q}}_{h, n}^{k}(t) \phi_{n}(x) \\
& =\sum_{n=1}^{N_{p}} \boldsymbol{Q}_{h}^{k}\left(t, x_{n}\right) l_{n}(x)=\sum_{n=1}^{N_{p}} \boldsymbol{Q}_{h, n}^{k}(t) l_{n}(x)
\end{aligned}
$$

where $\hat{\boldsymbol{Q}}_{h, n}^{k}$ and $\boldsymbol{Q}_{h, n}^{k}$ are unknown modal and nodal coefficients, respectively. The basis functions $\phi_{n}$ are chosen such that the solution $\boldsymbol{Q}_{h}^{k}$ belongs to the following approximation space,

$$
\mathbb{V}_{p}^{k}=\left\{v: v^{k} \in \mathbb{P}^{p}\left(\Omega^{k}\right), \forall \Omega^{k} \in \Omega\right\}
$$

where $\mathbb{P}^{p}\left(\Omega^{k}\right)$ are polynomials of degree at most $p=$ $N_{p}-1$ in each element $\Omega^{k}$. To rewrite Eq. (40) in compact form the continuous variables are replaced by the finite sum given by Eq. (41) and the (constant) local element matrices defined in section 3.1.2 are used to give

$$
\begin{aligned}
& \frac{d \boldsymbol{W}_{h}^{k}}{d t}= \frac{2}{\Delta x^{k}} \hat{\mathcal{M}}^{-1} \hat{\mathcal{S}}^{T} \boldsymbol{H}_{h}^{k}+\frac{2}{\Delta x^{k}} \hat{\mathcal{L}}\left[\begin{array}{c}
-\left.\boldsymbol{h}^{*}\right|_{x_{L}^{k}} \\
\left.\boldsymbol{h}^{*}\right|_{x_{R}^{k}}
\end{array}\right] \\
& \boldsymbol{G}_{h}^{k}=-\sqrt{D_{a}} \frac{2}{\Delta x^{k}} \hat{\mathcal{M}}^{-1} \hat{\mathcal{S}}^{T} \boldsymbol{C}_{h}^{k} \\
&-\frac{2}{\Delta x^{k}} \sqrt{D_{a}} \hat{\mathcal{L}}\left[\begin{array}{c}
-\left.\boldsymbol{c}^{*}\right|_{x_{L}^{k}} \\
\left.\boldsymbol{c}^{*}\right|_{x_{R}^{k}} ^{k}
\end{array}\right]
\end{aligned}
$$

where

$$
\begin{aligned}
\boldsymbol{H}_{h}^{k} & =\left(\boldsymbol{h}_{h}^{k}\left(x_{1}\right), \ldots, \boldsymbol{h}_{k}^{k}\left(x_{N_{p}}\right)\right)^{T} \\
\boldsymbol{G}_{h}^{k} & =\left(\boldsymbol{g}_{h}^{k}\left(x_{1}\right), \ldots, \boldsymbol{g}_{k}^{k}\left(x_{N_{p}}\right)\right)^{T} \\
\boldsymbol{C}_{h}^{k} & =\left(\boldsymbol{c}_{h}^{k}\left(x_{1}\right), \ldots, \boldsymbol{c}_{k}^{k}\left(x_{N_{p}}\right)\right)^{T}
\end{aligned}
$$

are nodal values defined on the domain $\Omega$ and the flux $\boldsymbol{h}_{h}^{k}$ is computed explicitly by

$$
\boldsymbol{h}_{h}^{k}=u \boldsymbol{c}_{h}^{k}-\sqrt{D_{a}} \boldsymbol{g}_{h}^{k}
$$

since we restrict the numerical flux $\boldsymbol{c}^{*}$ to depend on $\boldsymbol{c}_{h}^{k}$ only, e.g. the gradient variable $\boldsymbol{g}$ is a function of $\boldsymbol{c}$. In Eq. (43) the factor $2 / \Delta x^{k}$ is the inverse Jacobian of the local affine transformation,

$$
x^{k}(r)=x_{L}^{k}+0.5(1+r) \Delta x^{k}
$$

used to map the reference element $I$ to the physical element $\Omega^{k}$. Eq. (43) represents $N_{p}$ ODEs for $N_{p}$ unknowns in each local element. The global approximation is assembled as a direct sum of local contributions, e.g. $\boldsymbol{Q}_{h}=\bigoplus_{k=1}^{K} \boldsymbol{Q}_{h}^{k}$.

\subsubsection{Numerical fluxes}

To complete the scheme suitable numerical fluxes must be specified. We use the notation

$$
\{\{\vartheta\}\}=0.5\left(\vartheta^{\ominus}+\vartheta^{\oplus}\right), \quad[[\vartheta]]=0.5\left(\hat{\boldsymbol{n}}^{\ominus} \vartheta^{\ominus}+\hat{\boldsymbol{n}}^{\oplus} \vartheta^{\oplus}\right)
$$

for the average and jump at boundary interfaces $\partial \Omega^{k}$, respectively. In Eq. (49), the superscripts $\ominus$ and $\oplus$ denote the interior and exterior boundary values, respectively. The numerical flux $h^{*}$ is written as a convective and a diffusive flux. It is reasonable to consider these contributions separately due to the convective-diffusive nature of the problem under consideration. Thus,

$$
h^{*}=h_{\text {conv }}^{*}\left(c_{h}^{k, \ominus}, c_{h}^{k, \oplus}\right)+h_{\text {diff }}^{*}\left(g_{h}^{k, \ominus}, g_{h}^{k, \oplus}\right)
$$

The convective flux is chosen as the Lax-Friedrichs flux given by

$$
h_{i, \text { conv }}^{*}=u\left\{\left\{c_{i}^{k}\right\}\right\}+0.5 u\left[\left[c_{i}^{k}\right]\right]
$$

For the diffusive flux we choose an internal penalty flux ((Douglas and Dupont (1976); Douglas et al. (2002))),

$$
h_{i, \mathrm{diff}}^{*}=\sqrt{D_{a}}\left(\left\{\left\{g_{i}^{k}\right\}\right\}-\tau\left[\left[c_{i}^{k}\right]\right]\right)
$$

such that optimal convergence rates of order $p+1$ can be expected for all $p$. We note that choosing $\tau=0$ will simplify the internal penalty flux to a central flux and for $\tau>0$ the penalty term stabilizes the scheme by preventing large jumps in $c_{i}^{k}$. In our numerical experiments, we scale $\tau$ to the problem by

$$
\tau=\frac{p^{2}}{\Delta x}
$$

Finally, we specify the numerical flux $\sqrt{D_{a}} c_{i}^{*}$ as a central flux,

$$
\sqrt{D_{a}} c_{i}^{*}=\sqrt{D_{a}}\left\{\left\{c_{i}^{k}\right\}\right\}
$$

\subsubsection{Boundary conditions}

We impose boundary conditions weakly by exploiting a symmetry principle. The inlet Robin condition Eq. (6) is implemented by defining the combined convective and diffusive fluxes as

$$
\begin{aligned}
& h_{h}^{1, \oplus}\left(x_{L}^{1}\right)=-h_{h}^{1, \ominus}\left(x_{L}^{1}\right)+2\left(u c_{f}\right) \\
& \Rightarrow\left\{\left\{h_{h}^{1}\left(x_{L}^{1}\right)\right\}\right\}=u c_{f}
\end{aligned}
$$

such that the inlet condition is satisfied on average. The outlet Neumann condition Eq. (8) is implemented by defining the convective flux as

$$
\begin{aligned}
& h_{h, \text { conv }}^{K, \oplus}\left(x_{R}^{K}\right)=h_{h, \text { conv }}^{K, \ominus}\left(x_{R}^{K}\right) \\
& \Rightarrow\left\{\left\{h_{h, \text { conv }}^{K}\left(x_{R}^{K}\right)\right\}\right\}=h_{h, \text { conv }}^{K, \ominus}\left(x_{R}^{K}\right)
\end{aligned}
$$


Table 1: Coefficients for the low-storage 5-stage 4th-order ERK method (Carpenter and Kennedy (1994))

\begin{tabular}{cccc}
\hline$i$ & $a_{i}^{R K}$ & $b_{i}^{R K}$ & $c_{i}^{R K}$ \\
\hline 1 & 0 & $1432997174477 / 9575080441755$ & 0 \\
2 & $-567301805773 / 1357537059087$ & $5161836677717 / 13612068292357$ & $1432997174477 / 9575080441755$ \\
3 & $-2404267990393 / 2016746695238$ & $1720146321549 / 2090206949498$ & $2526269341429 / 6820363962896$ \\
4 & $-3550918686646 / 2091501179385$ & $3134564353537 / 4481467310338$ & $2006345519317 / 3224310063776$ \\
5 & $-1275806237668 / 842570457699$ & $2277821191437 / 14882151754819$ & $2802321613138 / 2924317926251$ \\
\hline
\end{tabular}

such that it is unmodified and the diffusive flux as

$$
h_{h, \text { diff }}^{K, \oplus}\left(x_{R}^{K}\right)=-h_{h, \text { diff }}^{K, \ominus}\left(x_{R}^{K}\right) \Rightarrow\left\{\left\{h_{h, \text { diff }}^{K}\left(x_{R}^{K}\right)\right\}\right\}=0
$$

such that it is zero on average.

\subsection{The total variation diminishing discontinuous Galerkin method}

The h-DG-TVD method is based on the hp-DG method using first-order polynomials in the approximation space. A post-processing procedure is applied after each stage of the ERK method (see section 3.4) to enforce the TVD property. In order to avoid destroying high-order accuracy in smooth regions of the solution, we apply a generalized slope limiter (Cockburn and Shu (2001)). We define the limited fluxes on the left and right element edge, respectively, as

$$
\begin{aligned}
& \varrho_{l}^{k}=\bar{c}_{h}^{k}-m\left(\bar{c}_{h}^{k}-c_{l}^{k}, \bar{c}_{h}^{k}-\bar{c}_{h}^{k-1}, \bar{c}_{h}^{k+1}-\bar{c}_{h}^{k}\right) \\
& \varrho_{r}^{k}=\bar{c}_{h}^{k}-m\left(\bar{c}_{r}^{k}-c_{h}^{k}, \bar{c}_{h}^{k}-\bar{c}_{h}^{k-1}, \bar{c}_{h}^{k+1}-\bar{c}_{h}^{k}\right)
\end{aligned}
$$

where $\bar{c}_{h}^{k}$ is the element average and we have defined the minmod function,

$$
m\left(y_{1}, y_{2}, y_{3}\right)= \begin{cases}s \min _{1 \leq n \leq 3}\left|y_{n}\right|, & \text { if }|s|=1 \\ 0, & \text { otherwise }\end{cases}
$$

with $s=1 / 3 \sum_{i=1}^{3} \operatorname{sign}\left(y_{i}\right)$. The generalized slope limiter is then applied to each element after each stage of the Runge Kutta ERK method using the following procedure:

- Compute $\varrho_{l}^{k}$ and $\varrho_{r}^{k}$ using Eqs. (58a) and (58b)

- if $\varrho_{l}^{k}=c_{h}^{k}\left(x_{l}^{k}\right)$ and $\varrho_{r}^{k}=c_{h}^{k}\left(x_{r}^{k}\right)$, there is no need for limiting and the local solution is not altered.

- if not, then limiting is needed and we compute a limited version of $c_{h}^{k}$ as $\Pi^{1} \tilde{c}_{h}^{k}$.

where $\tilde{c}_{h}^{k}$ is the linear approximation to $c_{h}^{k}$,

$$
\tilde{c}_{h}^{k}=\bar{c}_{h}^{k}+\left(x-x_{0}^{k}\right)\left(c_{h}^{k}\right)_{x}
$$

with $\left(c_{h}^{k}\right)_{x}$ the linear slope of element $k$ and $x_{0}$ represents the center coordinate of $\Omega^{k}$. On elements where limiting is required, we apply the following limiter:

$$
\Pi^{1} \tilde{c}_{h}^{k}=\bar{c}_{h}^{k}+\left(x-x_{0}^{k}\right) m\left(\left(c_{h}^{k}\right)_{x x}, \theta \frac{\bar{c}_{h}^{k+1}-\bar{c}_{h}^{k}}{\Delta x}, \theta \frac{\bar{c}_{h}^{k}-\bar{c}_{h}^{k-1}}{\Delta x}\right)
$$

where the classical minmod limiter is obtained for $\theta=1$ and the less restrictive, generalised minmod limiter for $\theta=2$ (Cockburn and Shu (2001)).

\subsection{The explicit Runge-Kutta method}

The semi-discrete schemes obtained after spatial discretization are of the form,

$$
\frac{d \boldsymbol{W}}{d t}=\boldsymbol{F}(t, \boldsymbol{W})
$$

We advance Eq. (62) in time using a 5-stage 4th-order low-storage ERK method (Carpenter and Kennedy (1994)) which requires storage and overwriting of only two vectors $\boldsymbol{v}_{j}$ and $\boldsymbol{\mu}_{j}$. The ERK method is given by the following steps: Set $\boldsymbol{\mu}_{0}=\boldsymbol{W}_{h}^{n}$, then for $j=1, \ldots, 5$ compute the intermediate functions:

$$
\begin{aligned}
& \boldsymbol{v}_{j}=a_{j}^{R K} \boldsymbol{v}_{j-1}+\Delta t \boldsymbol{F}\left(t_{n}+c_{j}^{R K} \Delta t, \boldsymbol{\mu}_{j-1}\right) \\
& \boldsymbol{\mu}_{j}=\boldsymbol{\mu}_{j-1}+b_{j}^{R K} \boldsymbol{v}_{j}
\end{aligned}
$$

The solution at the next time step is given by $\boldsymbol{W}_{h}^{n+1}=$ $\boldsymbol{\mu}_{5}$. Here, $\boldsymbol{W}_{h}^{n}=\boldsymbol{W}_{h}\left(t_{n}\right), \boldsymbol{W}_{h}^{n+1}=\boldsymbol{W}_{h}\left(t_{n+1}\right)$ and the fixed coefficients $a_{i j}^{R K}, b_{j}^{R K}$ and $c_{j}^{R K}$ are given in Table 1. After obtaining the solution of the total mass at the next time step $\boldsymbol{W}_{h}^{n+1}$ we apply Eq. (11) to obtain the concentrations in the mobile phase $\boldsymbol{C}_{h}^{n+1}=$ $\left(\boldsymbol{c}_{h}^{n+1}\left(x_{1}\right), \ldots, \boldsymbol{c}_{h}^{n+1}\left(x_{N_{p}}\right)\right)^{T}$.

In order to ensure linear stability of the ERK method, we constrain the time step as follows,

$$
\Delta t \leq C_{0} \frac{\min _{i}\left(\Delta x_{i}^{k}\right)}{u+2 D_{a} / \min _{i}\left(\Delta x_{i}^{k}\right)}
$$

for some constant $C_{0}$ of order 1 . 


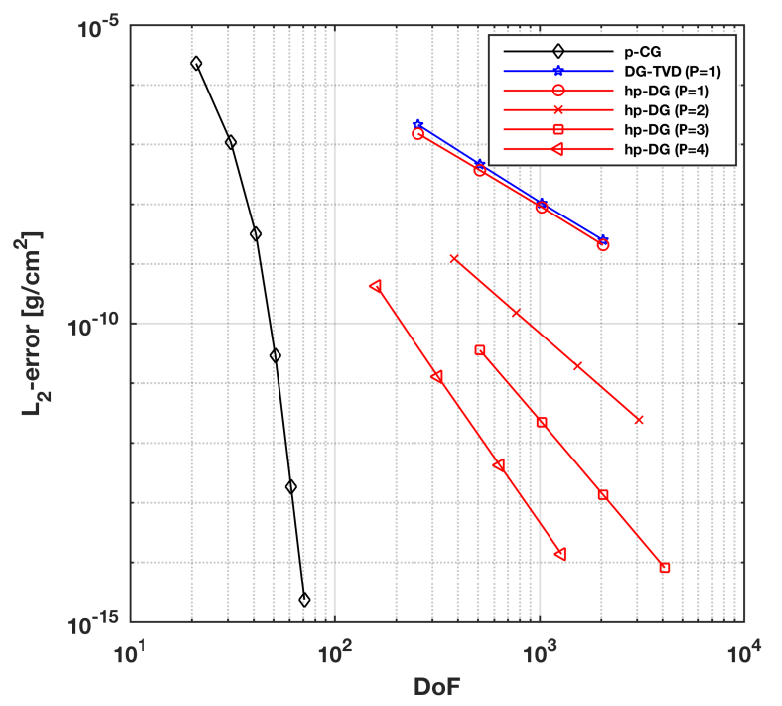

Figure 1: The error measured in the $L^{2}$ norm of the concentration band inside the column after advancing the problem in time from $T=2$ until $T=2.5$ as a function of degrees of freedom used in the spatial discretization. Shown in logarithmic scale.

\section{Numerical test study}

In this section, we perform numerical tests to evaluate the performance of the numerical methods presented in section 3 for both linear and non-linear chromatographic systems. The methods have been implemented in Matlab code and compiled on a Macbook Pro with a 2.7 GHz Intel Core i5 processor and 8 GB RAM.

Table 2: Simulation parameters used in the linear case study (section 4.1)

\begin{tabular}{lccc}
\hline Parameters & Symbol & Values & Unit \\
\hline Column length & $L$ & 1 & $\mathrm{~cm}$ \\
Porosity & $\epsilon$ & 0.4 & - \\
Interstitial velocity & $u$ & 0.46 & $\mathrm{~cm} \mathrm{~min}^{-1}$ \\
Dispersion Coefficient & $D_{a}$ & 0.002 & $\mathrm{~cm}^{2} \mathrm{~min}^{-1}$ \\
Henry's constant & $a$ & 1 & - \\
Feed concentration & $c_{f}$ & 1 & $\mathrm{~g} \mathrm{l}^{-1}$ \\
Injection time & $t_{\text {inj }}$ & 1 & $\mathrm{~min}$ \\
\hline
\end{tabular}

4.1. Case 1: Linear chromatography with smooth initial conditions

The designed spatial convergence of the numerical methods are validated experimentally by convergence tests on a single-component problem with a linear isotherm Eq. (3). The analytical solution to this problem is given by Qamar et al. (2013) for $0<t \leq t_{i n j}$ as

$$
c(t, z)=c_{\text {init }}+\left(c_{\text {inj }}-c_{\text {init }}\right) H(t, z)
$$

and by

$$
c(t, z)=c_{i n i t}+\left(c_{i n j}-c_{i n i t}\right) H(t, z)-c_{i n j} H\left(t-t_{i n j}, z\right)
$$

for $t>t_{i n j}$ with

$$
\begin{aligned}
& H(t, z)=\frac{1}{2} \operatorname{erfc}\left[\frac{\alpha z-t P e_{a}}{2 \sqrt{\alpha t}}\right]+\left(\frac{t P e_{a}^{2}}{\pi \alpha}\right)^{1 / 2} \\
& \times \exp \left[-\frac{\left(\alpha z-t P e_{a}^{2}\right)}{4 \alpha t}\right]-\frac{1}{2}\left(1+z P e_{a}+\frac{t P e_{a}^{2}}{\alpha}\right) \\
& \times \exp \left(z P e_{a}\right) \operatorname{erfc}\left[\frac{\alpha z+t P e_{a}}{2 \sqrt{\alpha t}}\right]+\left(\frac{4 t P e_{a}^{2}}{\pi \alpha}\right)^{1 / 2} \\
& \times\left[1+\frac{P e_{a}}{4}\left(2-z+\frac{t P e_{a}}{\alpha}\right)\right] \\
& \times \exp \left[P e_{a}-\frac{\alpha}{4 t}\left(2-z+\frac{t P e_{a}}{\alpha}\right)^{2}\right] \\
& -P e_{a}\left[\left(2-z+\frac{3 t P e_{a}}{2 \alpha}\right)+\frac{P e_{a}}{4}\left(2-z+\frac{t P e_{a}}{\alpha}\right)^{2}\right] \\
& \exp \left(P e_{a}\right) \operatorname{erfc}\left[\frac{\alpha(2-\alpha)+t P e_{a}}{2 \sqrt{\alpha t}}\right]
\end{aligned}
$$

Here, $z:=x / L$ is the normalized length, $P e_{a}:=L u / D_{a}$ is the apparent Peclet number and $\alpha:=(1+a F) P e_{a} L / u$ is a constant. We study a problem presented by Qamar et al. (2013) with detailed simulation parameters given in Table 2. Under these conditions, Qamar et al. (2013) used moment analysis to predict the elution of a normally distributed peak. After 2 min of simulation time, the problem is sufficiently smooth to expect experimental convergence rates equal to the theoretical values. We use the analytical solution to specify the column initial conditions after $2 \mathrm{~min}$ and advance the solution in time using our numerical methods from the exact initial conditions until the final time $t_{f}=2.5 \mathrm{~min}$. We measure the $L^{1}, L^{2}$ and $L^{\infty}$ errors over the spatial domain, defined respectively by

$$
\begin{aligned}
L^{1} \text {-error } & :=\int_{\Omega}|\boldsymbol{E}| d \Omega \\
L^{2} \text {-error } & :=\sqrt{\int_{\Omega} \boldsymbol{E}^{2} d \Omega} \\
L^{\infty} \text {-error } & :=\max _{i}\left|\boldsymbol{E}_{i}\right|
\end{aligned}
$$

where $\boldsymbol{E}=\left(\boldsymbol{e}_{1}, \ldots, \boldsymbol{e}_{N_{c}}\right)^{T}$ are errors evaluated at nodal points between the simulated and exact solution. We evaluate the integrals in Eqs. (68a) and (68b) using the nodal mass matrix given by Eq. (21a). 
Table 3: $\mathrm{L}^{1}, \mathrm{~L}^{2}\left(\mathrm{~g} / \mathrm{cm}^{2}\right)$ and $L^{\infty}\left(\mathrm{g} / \mathrm{cm}^{3}\right)$ errors and convergence rates for the linear problem using the hp-DG and h-DG-TVD methods

\begin{tabular}{|c|c|c|c|c|c|c|c|c|}
\hline Method & $K$ & $L^{1}$ error & $L^{2}$ error & $\mathrm{L}^{\infty}$ error & $L^{1}$ order & $L^{2}$ order & $L^{\infty}$ order & Theor. \\
\hline \multirow[t]{4}{*}{ hp-DG $\left(\mathbb{P}_{1}\right)$} & 32 & $1.49 \mathrm{E}-07$ & $1.52 \mathrm{E}-07$ & $7.99 \mathrm{E}-07$ & - & - & - & 2 \\
\hline & 64 & $3.66 \mathrm{E}-08$ & $3.70 \mathrm{E}-08$ & $1.95 \mathrm{E}-07$ & 2.03 & 2.04 & 2.03 & \\
\hline & 128 & $8.80 \mathrm{E}-09$ & $8.88 \mathrm{E}-09$ & $4.69 \mathrm{E}-08$ & 2.06 & 2.06 & 2.06 & \\
\hline & 256 & $2.03 \mathrm{E}-09$ & $2.07 \mathrm{E}-09$ & $1.10 \mathrm{E}-08$ & 2.11 & 2.10 & 2.09 & \\
\hline \multirow[t]{5}{*}{ hp-DG $\left(\mathbb{P}_{2}\right)$} & 32 & $5.16 \mathrm{E}-10$ & $1.22 \mathrm{E}-09$ & $1.03 \mathrm{E}-08$ & - & - & - & \\
\hline & 64 & $5.73 \mathrm{E}-11$ & $1.52 \mathrm{E}-10$ & $1.14 \mathrm{E}-09$ & 3.17 & 3.00 & & \\
\hline & 128 & $6.78 \mathrm{E}-12$ & $1.93 \mathrm{E}-11$ & $1.35 \mathrm{E}-10$ & 3.08 & 2.98 & & \\
\hline & 256 & $8.26 \mathrm{E}-13$ & $2.43 \mathrm{E}-12$ & $1.65 \mathrm{E}-11$ & 3.04 & 2.99 & & \\
\hline & & & - & & & & & \\
\hline \multirow[t]{5}{*}{ hp-DG $\left(\mathbb{P}_{3}\right)$} & 32 & $2.80 \mathrm{E}-11$ & $3.56 \mathrm{E}-11$ & $2.86 \mathrm{E}-10$ & - & - & & 4 \\
\hline & 64 & $1.73 \mathrm{E}-12$ & $2.21 \mathrm{E}-12$ & $1.90 \mathrm{E}-11$ & 4.02 & 4.01 & 3.91 & \\
\hline & 128 & $1.04 \mathrm{E}-13$ & $1.34 \mathrm{E}-13$ & $1.19 \mathrm{E}-12$ & 4.05 & 4.04 & 3.99 & \\
\hline & 256 & $6.18 \mathrm{E}-15$ & $8.15 E-15$ & $7.48 \mathrm{E}-14$ & 4.07 & & 4.00 & \\
\hline & & - & & & & & & \\
\hline \multirow[t]{5}{*}{ hp-DG $\left(\mathbb{P}_{4}\right)$} & 16 & $2.28 \mathrm{E}-10$ & $4.26 \mathrm{E}-10$ & $4.42 \mathrm{E}-09$ & & & - & 5 \\
\hline & 32 & $5.58 \mathrm{E}-12$ & $1.28 \mathrm{E}-11$ & $1.34 \mathrm{E}-10$ & 5.35 & 5.05 & 5.04 & \\
\hline & 64 & $1.64 \mathrm{E}-13$ & $4.19 \mathrm{E}-13$ & $4.32 \mathrm{E}-12$ & 5.09 & 4.94 & 4.95 & \\
\hline & 128 & $5.31 \mathrm{E}-15$ & $1.37 \mathrm{E}-14$ & $1.47 \mathrm{E}-13$ & 4.95 & 4.93 & 4.88 & \\
\hline & & - & & & & & & \\
\hline \multirow[t]{4}{*}{ h-DG-TVD $\left(\mathbb{P}_{1}\right)$} & 32 & $1.69 \mathrm{E}-07$ & $2.14 \mathrm{E}-07$ & $1.29 \mathrm{E}-06$ & - & - & - & 2 \\
\hline & 64 & $4.00 \mathrm{E}-08$ & $4.65 \mathrm{E}-08$ & $3.89 \mathrm{E}-07$ & 2.08 & 2.20 & 1.73 & \\
\hline & 128 & $9.60 \mathrm{E}-09$ & $1.04 \mathrm{E}-08$ & $5.56 \mathrm{E}-08$ & 2.06 & 2.16 & 2.81 & \\
\hline & 256 & $2.39 \mathrm{E}-09$ & $2.50 \mathrm{E}-09$ & $1.51 \mathrm{E}-08$ & 2.01 & 2.06 & 1.88 & \\
\hline
\end{tabular}

The computed solution is compared to the analytical solution. Results using the p-CG, hp-DG with $p \in[1,4]$ and h-DG-TVD for different mesh resolutions are shown in Fig. 1 and Table 3. The time step size was in this case chosen sufficiently small not to affect the accuracy of the simulations. From Table 1, we conclude that the experimental convergence rate is $p+1$ for the hp-DG method while the h-DG-TVD method is second-order accurate. This gives confidence that our numerical methods are correctly implemented, and that both the temporal and spatial discretizations are working as expected. From Fig. 1 it is clear that the p-CG method has the superior convergence rate on this problem, achieving spectral (exponential) convergence. In Fig. 2 we show the numerical solution at the final time $t_{f}=2.5$ obtained using (a) hp-DG $\left(\mathbb{P}_{1}\right)$, (b) hp-DG $\left(\mathbb{P}_{2}\right)$, (c) h-DG-TVD and (d) p-CG using $30 \mathrm{DoF}$ in the spatial discretization together with the analytical solution. The results shows that the injected concentration profile has evolved into a normally distributed peak shape as expected. The solution obtained using $\mathrm{p}-\mathrm{CG}$ is sufficiently resolved, the solution by hp-DG $\left(\mathbb{P}_{2}\right)$ is slightly unresolved while the solutions obtained using both hp-DG $\left(\mathbb{P}_{1}\right)$ and h-DG-TVD are unresolved on this grid.

Table 4: Simulation parameters for the non-linear case study (section 4.2).

\begin{tabular}{llll}
\hline Parameters & Symbol & Values & Unit \\
\hline Column length & $L$ & 1 & $\mathrm{~cm}$ \\
Porosity & $\epsilon$ & 0.4 & - \\
Interstitial velocity & $u$ & 1 & $\mathrm{~cm} \mathrm{~min}^{-1}$ \\
Constants used in Eq. (2) & $\boldsymbol{a}$ & $(0.5,1)^{T}$ & - \\
Constants used in Eq. (2) & $\boldsymbol{b}$ & $(0.05,0.01)^{T}$ & $\mathrm{lg}^{-1}$ \\
Feed concentrations & $\boldsymbol{c}_{f}$ & $(10,10)^{T}$ & $\mathrm{~g} \mathrm{l}^{-1}$ \\
\hline
\end{tabular}

\subsection{Case 2: Two-component nonlinear chromatogra- phy}

For non-linear models, no analytical solutions are available. Therefore, we rely on numerical techniques to obtain solutions. On the basis of the obtained accurate results in the previous linear case, we expect that 


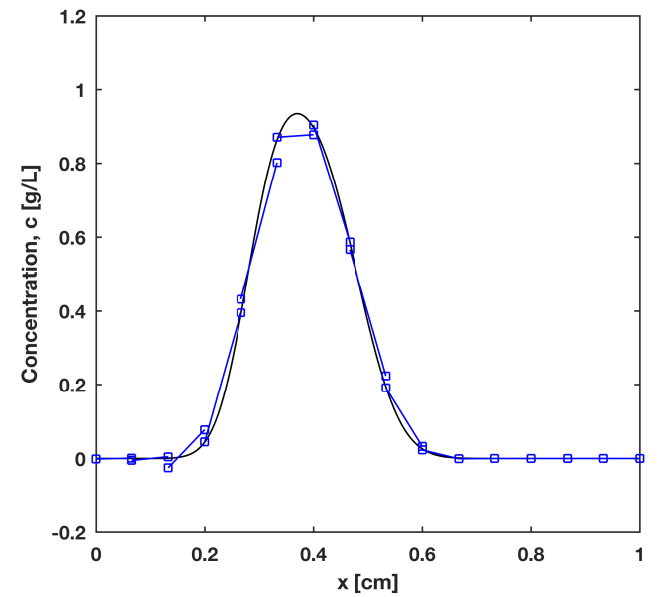

(a) hp-DG $\left(\mathbb{P}_{1}\right)$, DOF $=30$

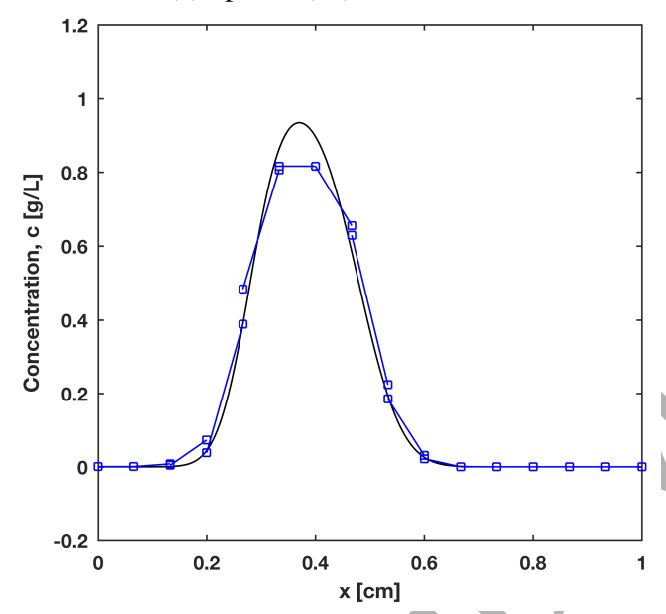

(c) h-DG-TVD, DOF $=30$

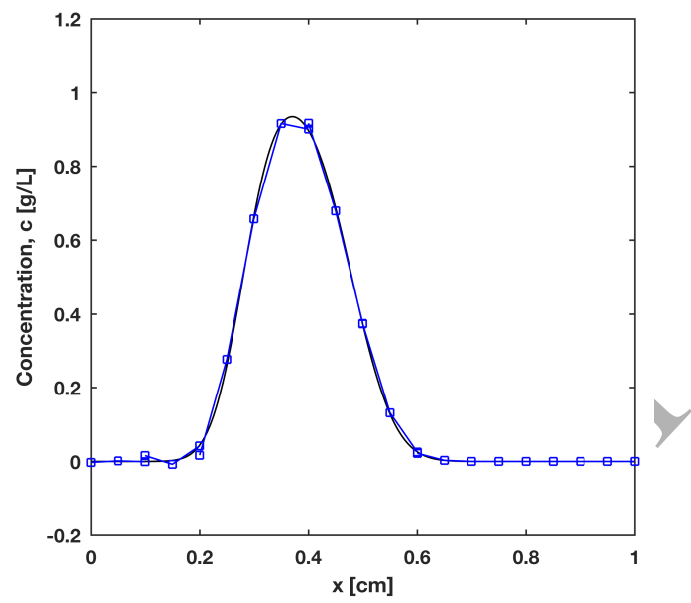

(b) hp-DG $\left(\mathbb{P}_{2}\right)$, DOF $=30$

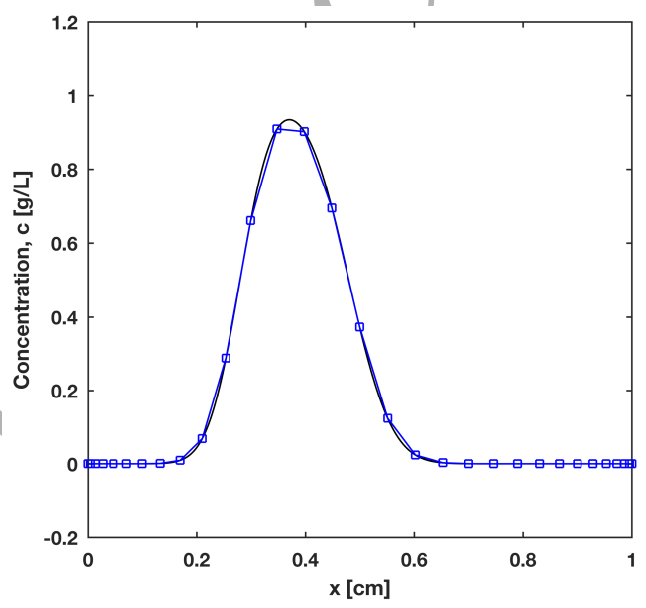

(d) $\mathrm{p}-\mathrm{CG}\left(\mathbb{P}_{29}\right), \mathrm{DOF}=30$

Figure 2: Simulation of the linear case. The solid black line is the exact solution and the blue squares are nodal values of the numerical approximations.

the proposed methods could prodúce accurate results for non-linear models as well. We consider a non-linear problem studied by Shipilova et al. (2008) where two components compete for available slots on the stationary phase according to the multicomponent Langmuir isotherm given by Eq. (2). The detailed simulation parameters are given in Table 4. A rectangular pulse of the two-component liquid mixture is injected at the column inlet for $1.2 \mathrm{~min}$. In the following, we simulate the problem in a column of modest and high efficiency, respectively.

\subsubsection{Column of modest efficiency}

The problem is simulated using a low number of apparent theoretical plates of $N_{a}=500$ to test the per- formance of the numerical methods under typical liquid chromatography conditions. Fig. 3 shows the simulation results obtained using $\mathrm{p}-\mathrm{CG}, \mathrm{hp}-\mathrm{DG}\left(\mathbb{P}_{3}\right)$ and h-DG-TVD using two different mesh resolutions. The numerical results obtained with $\mathrm{p}-\mathrm{CG}$ included no stabilization. A highly resolved reference solution was obtained using a 9th-order hp-DG method with 100 elements (1000 DoF).

The simulation results are shown in Fig. 3 and are compared with the highly resolved reference solution (black line). Despite the low number of apparent theoretical plates, the reference solution includes rather steep concentration fronts. Since we are integrating the solution with high-precision in the spatial discretization due to the use of high-order methods the solution is fully 
resolved leading to steep concentration fronts, even for low plate numbers. It might seem surprising that the elution profile of the first component 'shoots over' the injected concentration of $10 \mathrm{~g} / \mathrm{L}$. This phenomena is however well-known in non-linear liquid chromatographic processes and is a consequence of displacement effects. This provides evidence that our codes are working as expected.

In Fig. 3(b) we observe that the solution by the pCG method has converged towards the reference solution using just 62 DoF in the spatial discretization (there is no visual difference between the simulated and reference solution). When using the $\mathrm{p}-\mathrm{CG}$ method on an insufficiently resolved grid with 22 DoFs, the solution oscillates (see Fig. 3(a)). These oscillations do not indicate that the method is unstable, and they are removed simply by adding more resolution. In the following, we compare the convergence properties of the hpDG and the h-DG-TVD methods with the p-CG method by visual inspection. Both methods are applied on the same problem using 62 DoF in the spatial discretization which resulted in a sufficiently resolved solution when the p-CG method was applied. The results of the simulations are shown in Fig. 3(d) and (f) using the hp-DG $\left(\mathbb{P}_{3}\right)$ method and the h-DG-TVD method, respectively. By visual inspection, the hp-DG $\left(\mathbb{P}_{3}\right)$ method provides an oscillatory solution while the solution by the h-DGTVD method is artificially dispersed. Hence, in order to obtain accurate solutions, both methods must be applied with a larger amount of DoF in the spatial discretization compared to when the p-CG method was used. This indicates, that the p-CG method has the superior convergence properties on this problem (since it converges using the fewest DoFs). Fig. 3(c) and (d) shows, that the hp-DG method provides oscillatory/solutions when applied with an insufficient resolution. Again, this does not indicate that the method is unstable, merely that an insufficient resolution was applied. The oscillations are removed simply by adding enough resolution. The only method that does not oscillate on an under-resolved grid is the h-DG-TVD method (see Fig. 3(e) and (f)). However, it comes at the high cost of reducing the accuracy to first order in some parts of the solution such that many DoFs are required to obtain fully resolved solutions. Since the hp-DG method is already stable for the simple linear fluxes used to model liquid chromatography applications without applying any stabilization, the use of a limiter to enforce additional (unnecessary) stabilization seems to only worsen the convergence properties of the hp-DG method. Therefore, we do not recommend solving non-linear liquid chromatography models under liquid chromatography conditions using shock-
Table 5: Computational cost for a fixed accuracy measured by the $\mathrm{L}^{1}$ error $\left(\operatorname{min~} \mathrm{g}^{-1}\right)$ for the non-linear case (Table 4) using $N_{a}=500$ apparent theoretical plates.

\begin{tabular}{llllll}
\hline Scheme & $\mathrm{L}_{T}^{1}$ error & $\Delta t(\mathrm{~min})$ & DoFs & $\mathrm{CPU}(\mathrm{s})$ & CPU $_{\text {ratio }}$ \\
\hline p-CG $\left(\mathbb{P}_{61}\right)$ & $1.08 \mathrm{E}-02$ & $1.6 \mathrm{E}-03$ & 62 & 3.9 & 1 \\
hp-DG $\left(\mathbb{P}_{8}\right)$ & $1.10 \mathrm{E}-02$ & $1.6 \mathrm{E}-03$ & 108 & 5.8 & 1.5 \\
hp-DG $\left(\mathbb{P}_{3}\right)$ & $1.10 \mathrm{E}-02$ & $1.6 \mathrm{E}-03$ & 148 & 6.3 & 1.6 \\
hp-DG $\left(\mathbb{P}_{2}\right)$ & $1.11 \mathrm{E}-02$ & $1.6 \mathrm{E}-03$ & 195 & 7.2 & 1.9 \\
hp-DG $\left(\mathbb{P}_{1}\right)$ & $1.07 \mathrm{E}-02$ & $1.6 \mathrm{E}-03$ & 500 & 24.4 & 6.2 \\
h-DG-TVD $\left(\mathbb{P}_{1}\right)$ & $1.12 \mathrm{E}-02$ & $3.0 \mathrm{E}-04$ & 1000 & 414.0 & 67.9 \\
\hline
\end{tabular}

capturing methods. Instead, we suggest (based on the presented results) to consider arbitrary high-order methods.

Having investigated the convergence properties of the methods, we now study the the amount of work required to achieve a given level of accuracy. To this purpose, we compare the simulation results with the highly resolved reference solution and measure the $L^{1}$ errors over the temporal domain at the outlet of the column by

$$
L_{T}^{1} \text {-error }=\int_{T}|\boldsymbol{E}| d t
$$

where $T \in\left[0, t_{f}\right]$ is the temporal domain and $\boldsymbol{E}=$ $\left(\boldsymbol{e}_{1}, \ldots, \boldsymbol{e}_{N_{c}}\right)^{T}$ are nodal errors between the simulated and the reference solution. The integral in Eq. (69) is evaluated using the trapezoidal method. We evaluate the performance of the three methods based on how much work is required in terms of CPU time to achieve an $L_{T}^{1}$-error of atleast $0.01 \mathrm{~min} \times \mathrm{g} / 1$ (at this point we cannot tell the difference between the simulated and the reference solutions by visual inspection). In Table 5 we present the simulation results, showing that the $\mathrm{p}-\mathrm{CG}$ method is the most efficient method for this problem in terms of both CPU time and discrete problem size. The hp-DG method becomes more efficient when using polynomials of higher order, although the effect seems to become less obvious after using more than third-order polynomials. The h-DG-TVD method is inefficient on this problem compared to the other methods. The limiter is activated whenever an oscillation is detected, reducing the accuracy to first-order in this element. Therefore, it requires two times more DoFs compared to the second-order accurate hp-DG $\left(\mathbb{P}_{1}\right)$ method. Further, its stable time step size was more restrictive due to the use of many DoFs.

\subsubsection{Column of high efficiency}

We now study the simulation of the problem in a very efficient column with $N_{a}=3000$ apparent theoretical plates to test the robustness of the numerical methods when dispersion is practically absent leading to strong 


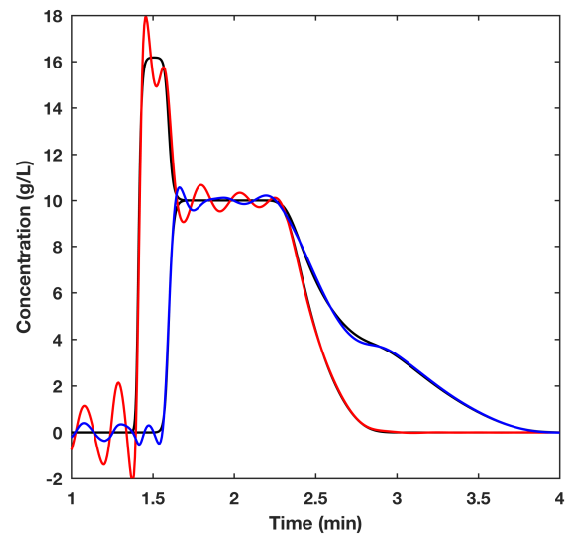

(a) $\mathrm{p}-\mathrm{CG}\left(\mathbb{P}_{21}\right), \mathrm{DoF}=22$

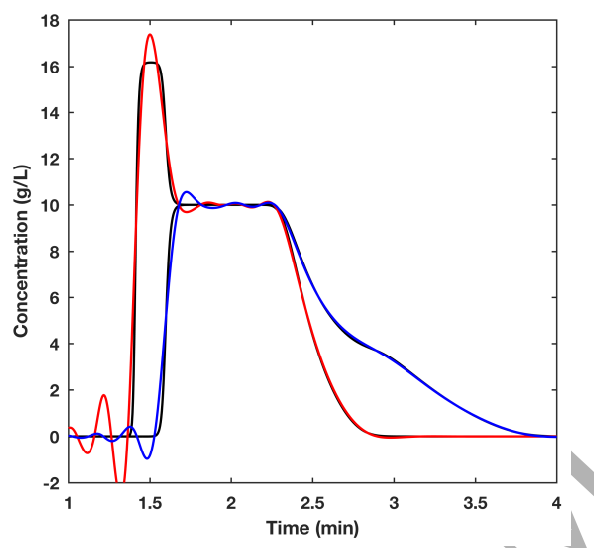

(c) hp-DG $\left(\mathbb{P}_{3}\right)$, DoF $=24$

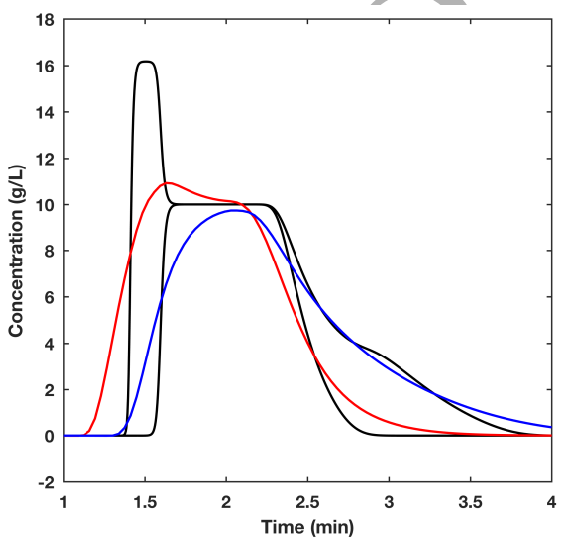

(e) h-DG-TVD, $D o F=22$

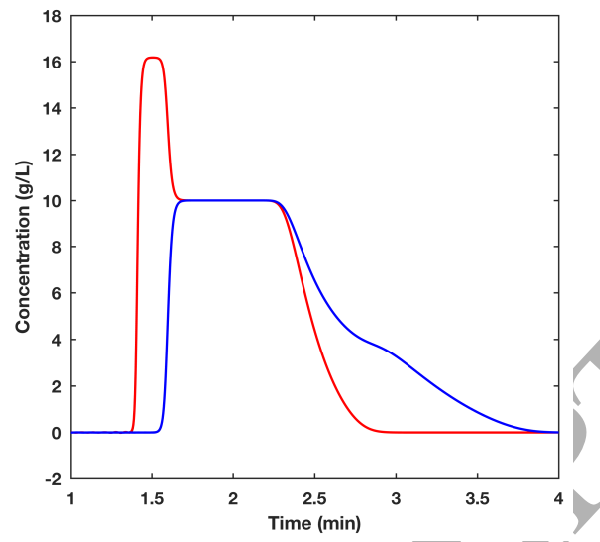

(b) $\mathrm{p}-\mathrm{CG}\left(\mathbb{P}_{61}\right), D o F=62$

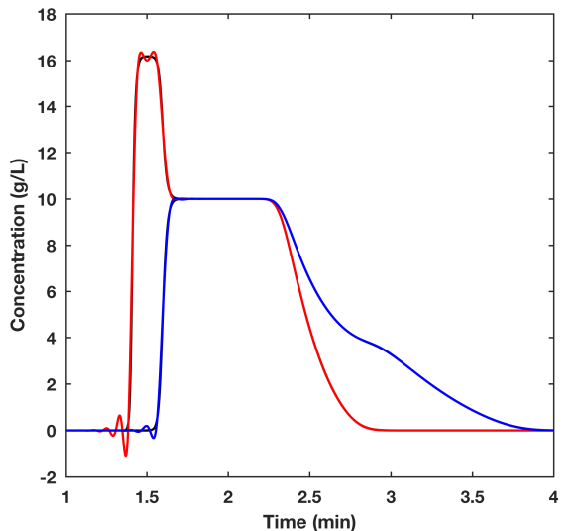

(d) hp-DG $\left(\mathbb{P}_{3}\right)$, DoF $=64$

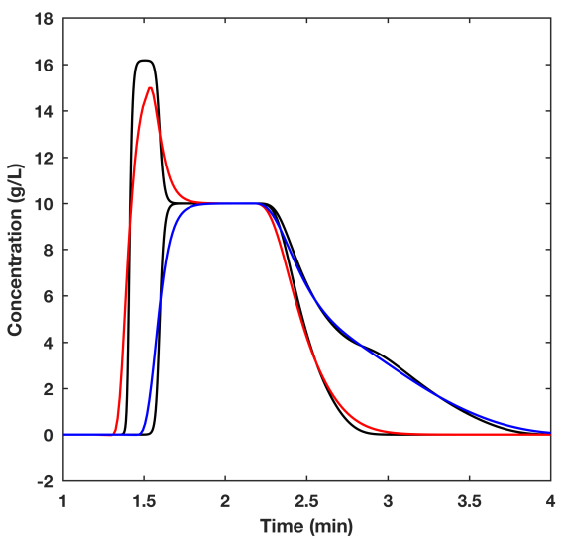

(f) h-DG-TVD, $D o F=62$

Figure 3: Simulation of the two component nonlinear case $\left(N_{a}=500\right)$. The solid black line is the fully resolved reference solution. The red and blue lines are numerical approximations for component one and two, respectively.

shock waves. In Fig. 4 we show the results of the simulations obtained using the p-CG, the hp-DG and the hDG-TVD methods on two grids of different resolution. In this case, we applied a mild filter to stabilize the p-
CG method, e.g. we apply an 8th order $(s=8)$ filter to $10 \%$ of the higest modes in the basis. That is, if we use a 100th-order polynomial, then we apply the modal filter with $i_{c}=90$. We applied the hp-DG method with no 


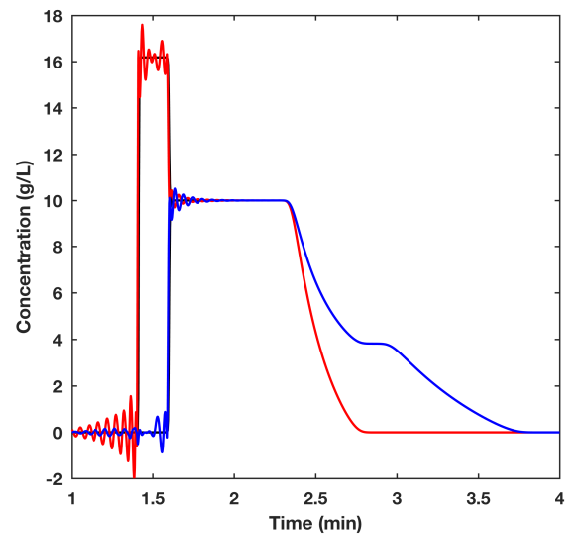

(a) $\mathrm{p}-\mathrm{CG}\left(\mathbb{P}_{99}\right), D o F=100$

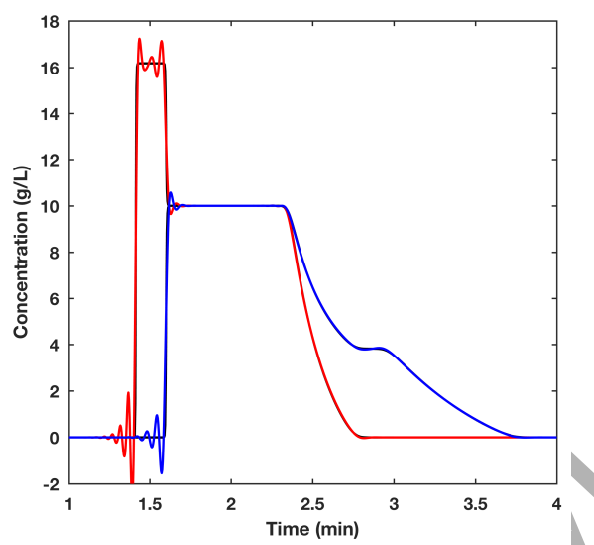

(c) hp-DG $\left(\mathbb{P}_{3}\right)$, DoF $=100$

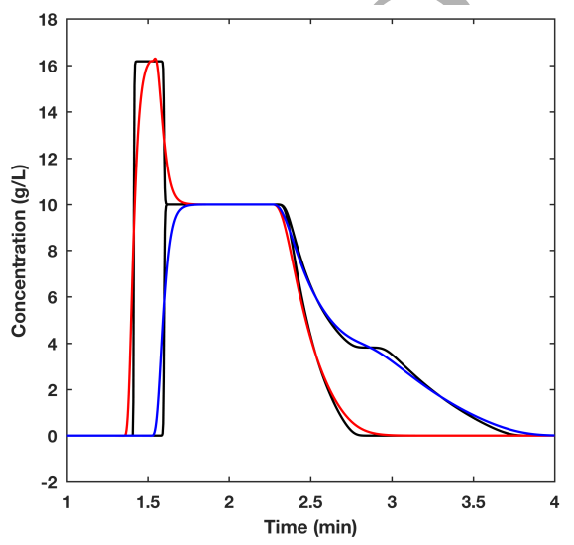

(e) h-DG-TVD, $D o F=100$

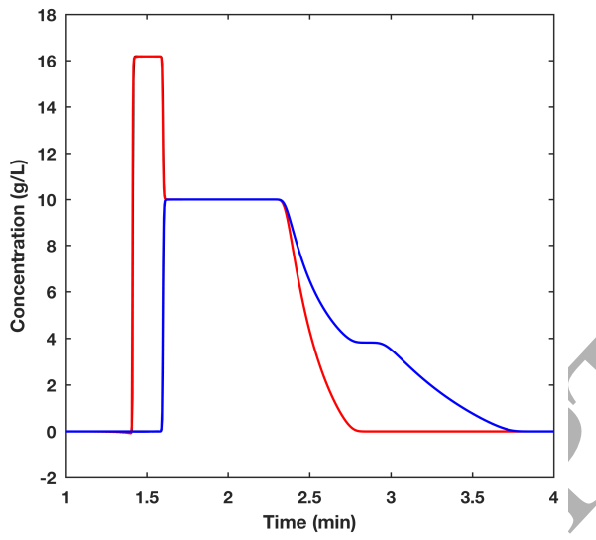

(b) $\mathrm{p}-\mathrm{CG}\left(\mathbb{P}_{209}\right), \mathrm{DoF}=210$

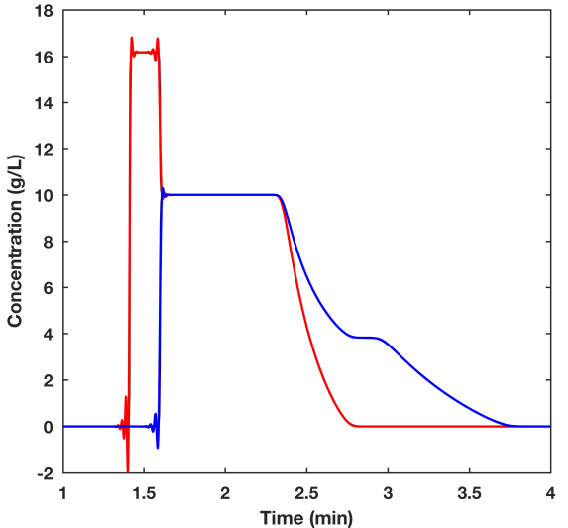

(d) hp-DG $\left(\mathbb{P}_{3}\right), D o F=208$

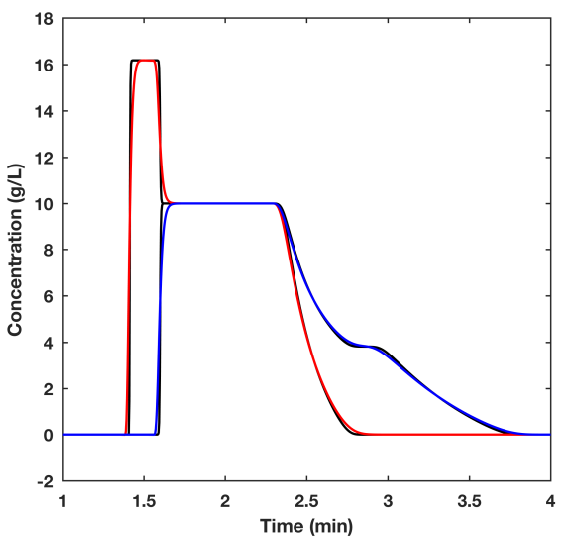

(f) h-DG-TVD, $D o F=210$

Figure 4: Simulation of the two component nonlinear case $\left(N_{a}=3000\right)$. The solid black line is the fully resolved reference solution. The red and blue lines are numerical approximations for component one and two, respectively.

stabilization, since the numerical upwind fluxes already provide enough dissipation of high-frequency modes to prevent instabilities. A fully resolved reference solution was obtained using a 9th-order hp-DG method with 300 elements (3000 DoF) for comparison with our simulations. In Fig 4 the reference solution is depicted with a solid black line. We note that the problem is challenging to solve for a numerical method due to the presence 
of strong shock-waves. In our simulations, we obtained shock-waves already after applying a column with just $N_{a}=3000$ apparent plates. We therefore have no reason to increase it further. The steepness of the concentration fronts can be compared with those reported by Shipilova et al. (2008); Javeed et al. (2013) where $N_{a}=5000$ apparent plates was used.

Comparing Fig. 4(b), (d) and (f), we see that it is again the $\mathrm{p}-\mathrm{CG}$ method which has the superior convergence properties, even in the presence of strong shock waves. In Fig. 4(a), (c) and (d) we demonstrate that both the p-CG and the hp-DG methods are oscillating on under-resolved grids, although both methods are stable. The oscillations are localized near the shock-waves while in smooth regions the solution is accurate. In Fig. 4(e) and (f) we demonstrate that the h-DG-TVD method is capable of providing non-oscillatory solutions on under-resolved grids.

Albeit the convergence properties of the $\mathrm{p}-\mathrm{CG}$ method is impressive, the decisive criterion for choosing between different numerical strategies is the cost required to obtain accurate solutions. We simulate the problem using the three methods until an $L_{T}^{1}$ error of 0.05 has been achieved (at this point the solution has converged by visual inspection). In Table 6 we show the simulation results, demonstrating that the $\mathrm{p}-\mathrm{CG}$ method is faster compared to the hp-DG method using first and second order polynomials. When using the hpDG method with third order polynomials the cost is similar. However, when using the hp-DG method with more than third-order polynomials, it becomes slightly faster. While the hp-DG method using $p>3$ is faster, the p-CG method still has the superior convergence properties resulting in a smaller discrete problem size. A small discrete problem size is essential when considering e.g. dynamic optimization applications or state-estimation algorithms. Holmqvist and Magnusson (2016) found for the solution of open-loop optimal control problems that a finite volume scheme with a WENO limiter was computationally advantageous over other finite volume discretizations using TVD limiters, although the finite volume WENO scheme required several times more CPU time. However, the WENO scheme required less finite volume elements to spatially resolve smooth parts of the solution, which significantly reduced the size of the considered dynamic optimization problem. Thus, although the hp-DG method is faster, the p-CG method is to prefer for such applications. The p-CG method is about 10 times faster compared to the h-DG-TVD method, even for problems including strong shock-waves. Hence, we suggest to use arbitrary high-order methods for cost-efficient simula-
Table 6: Computational cost for a fixed accuracy measured by the $\mathrm{L}^{1}$ error $\left(\operatorname{min~} \mathrm{gl}^{-1}\right)$ for the non-linear case (Table 4) using $N_{a}=3000$ apparent theoretical plates.

\begin{tabular}{llllll}
\hline Scheme & $\mathrm{L}^{1}$ error & $\Delta t(\mathrm{~min})$ & DoFs & CPU $(\mathrm{s})$ & CPU $_{\text {ratio }}$ \\
\hline p-CG $\left(\mathbb{P}_{210}\right)$ & $50.82 \mathrm{E}-03$ & $1.0 \mathrm{E}-04$ & 211 & 134.0 & 1.00 \\
hp-DG $\left(\mathbb{P}_{8}\right)$ & $51.42 \mathrm{E}-03$ & $1.0 \mathrm{E}-04$ & 279 & 119.6 & 0.89 \\
hp-DG $\left(\mathbb{P}_{3}\right)$ & $50.48 \mathrm{E}-03$ & $1.0 \mathrm{E}-04$ & 308 & 130.0 & 0.97 \\
hp-DG $\left(\mathbb{P}_{2}\right)$ & $50.35 \mathrm{E}-03$ & $1.0 \mathrm{E}-04$ & 348 & 166.4 & 1.24 \\
hp-DG $\left(\mathbb{P}_{1}\right)$ & $50.53 \mathrm{E}-03$ & $1.0 \mathrm{E}-04$ & 520 & 254.0 & 1.90 \\
h-DG-TVD $\left(\mathbb{P}_{1}\right)$ & $50.74 \mathrm{E}-03$ & $1.0 \mathrm{E}-04$ & 1040 & 1297.0 & 9.7 \\
\hline
\end{tabular}

tion of highly convection-dominated non-linear liquid chromatography processes instead of the second-order shock-capturing methods often used.

\section{Conclusions}

In this paper, we have compared several state-of-theart methods for simulation of linear and non-linear liquid chromatographic processes using columns of both low and high efficiency. The computational efficiency of the methods is determined with reference to a fully converged simulation of high spatial resolution for nonlinear processes. For linear processes, an analytical solution is available on which we analysed experimental convergence rates. We observed that a considerable saving in computational cost can be achieved by employing stable high-order methods compared to the second order (low-order) shock-capturing methods which are often used by default for simulation of liquid chromatography models. Furthermore, we have for the first time demonstrated, that the use of the continuous Galerkin spectral methods in combination with state-of-the-art spectral filtering techniques are highly suitable for efficient and accurate simulation of liquid chromatographic models.

The stable high-order methods presented here have been shown to oscillate on unresolved grids. While this is of less concern when considering Langmuir-type isotherms, it may become problematic for isotherms considering multipoint binding, e.g. the steric-massaction isotherm (Brooks and Cramer (1992)) used to model ion-exchange chromatography. Here, numerical under/overshoots can lead to ill-posed solutions leading to a shut-down of the computer program. Therefore, the presented high-order methods should be used on sufficiently resolved grids to obtain non-oscillatory solutions when considering isotherms that support multipoint binding. On the other hand, the total variation diminishing discontinuous Galerkin method will suppress the numerical oscillations, even on unresolved grids, at the cost of producing an overly dispersed solution. 


\section{Acknowledgement}

The project received financial support from Innovation Fund Denmark through the BIOPRO2 strategic research centre (Grant number 4105-00020B)

\section{References}

Bassi, F., Rebay, S., 1997. A high-order accurate discontinuous finite element method for the numerical solution of the compressible Navier-Stokes equations. Journal of Computational Physics 131, 267-279.

Baumann, P., Hahn, T., Hubbuch, J., 2015. High-throughput microscale cultivations and chromatography modeling: Powerful tools for integrated process development. Biotechnology and Bioengineering 112 (10), 2123-2133.

Brooks, C. A., Cramer, S. M., 1992. Steric mass-action ion exchange: Displacement profiles and induced salt gradients. AIChE Journal 38 (12), 1969-1978.

Brown, S. F., Ogden, M. D., Fraga, E. S., 2018. Efficient simulation of chromatographic separation processes. Computers and Chemical Engineering 110, 69-77.

Carpenter, M., Kennedy, C., 1994. Fourth-order 2n-storage rungekutta schemes. NASA-TM-109112, 1-24.

Cockburn, B., Shu, C.-W., 2001. Runge-kutta discontinuous galerkin methods for convection-dominated problems. Journal of Scientific Computing 16 (3), 173-261.

Danckwerts, P., 1953. Continuous flow systems. Chemical Engineering Science 2 (1), 1-13.

Donat, R., Guerrero, F., Mulet, P., 2018. ImplicitExplicit WENO scheme for the equilibrium dispersive model of chromatography. Applied Numerical Mathematics 123, 22-42.

Douglas, J., Dupont, T., 1976. Interior penalty procedures for elliptic and parabolic Galerkin methods. In: Glowinski, R., Lions, J. (Eds.), Computing Methods in Applied Sciences. Lecture Notes in Physics. Vol. 58. Springer, Berlin, Heidelberg, pp. 207-216.

Douglas, N., Brezzi, F., Cockburn, B., Marini, L., 2002. Unified analysis of discontinuous Galerkin methods for elliptic problems. Siam Journal on Numerical Analysis 39 (5), 1749-1779.

Gottlieb, D., Hesthaven, J. S., 2001. Spectral methods for hyperbolic problems. Journal of Computational and Applied Mathematics $128(1-2), 83-131$.

Gottlieb, D., Shu, C.-W., 1997. On the Gibbs Phenomenon and Its Resolution. SIAM Review 39 (4), 644-668.

Guiochon, G., Felinger, A., Shirazi, D. G. G., 2006. Fundamentals of preparative and nonlinear chromatography, 2nd Edition. Elsevier.

Harten, A., Engquist, B., Osher, S. Chakravarthy, S. R., 1987. Uniformly High Order Accurate Essentially Non-oscillatory Schemes, III. Journal of Computational Physics 71 (2), 231-303.

Hesthaven, J. S., Kirby, R. M., 2008. Filtering in legendre spectral methods. Mathematics of Computation 77 (263), 1425-1452.

Hesthaven, J. S., Warburton, T., 2002. Nodal high-order methods on unstructured grids - I. Time-domain solution of Maxwell's equations. Journal of Computational Physics 181 (1), 186-221.

Hesthaven, J. S., Warburton, T., 2008. Nodal Discontinuous Galerkin Methods: Algorithms, Analysis, and Applications. Springer, New York.

Holmqvist, A., Magnusson, F., 2016. Open-loop optimal control of batch chromatographic separation processes using direct collocation. Journal of Process Control 46, 55-74.

Huuk, T. C., Hahn, T., Osberghaus, A., Hubbuch, J., 2014. Modelbased integrated optimization and evaluation of a multi-step ion exchange chromatography. Separation and Purification Technology $136,207-222$.
Javeed, S., Qamar, S., Ashraf, W., Warnecke, G., Seidel-Morgenstern, A., 2013. Analysis and numerical investigation of two dynamic models for liquid chromatography. Chemical Engineering Science 90, 17-31.

Javeed, S., Qamar, S., Seidel-Morgenstern, A., Warnecke, G., 2011a. A discontinuous Galerkin method to solve chromatographic models. Journal of Chromatography A 1218 (40), 7137-7146.

Javeed, S., Qamar, S., Seidel-Morgenstern, A., Warnecke, G., 2011 b. Efficient and accurate numerical simulation of nonlinear chromatographic processes. Computers and Chemical Engineering 35 (11), 2294-2305.

Kelley, C., 2003. Solving Nonlinear Equations with Newtons Method. SIAM.

Kopriva, D. A., 2009. Implementing spectral methods for partial differential equations. Springer Netherlands.

Lim, Y.-I., Chang, S.-C., Jørgensen, S. B., 2004, A novel partial differential algebraic equation (PDAE) solver: Iterative space-time conservation element/solution element (CE/SE) method. Computers and Chemical Engineering 28 (8), 1309-1324.

Ma, Z., Guiochon, G., 1992. Shock layer thickness in the case of wide bands of single components and binary mixtures in non-linear liquid chromatography. Journal of Chromatography A 609 (1-2), 1933.

Medi, B., Amanullah, M., 2011. Application of a finite-volume method in the simulation of chromatographic systems: Effects of flux limiters. Industrial and Engineering Chemistry Research 50 (3), 1739-1748.

Meyer, K., Huusom, J., Abildskov, J., 2018. High-order approximation of chromatographic models using a nodal discontinuous Galerkin approach. Computers and Chemical Engineering 109, 68-76.

Pirrung, S. M., van der Wielen, L. A., van Beckhoven, R. F., van de Sandt, E. J., Eppink, M. H., Ottens, M., 2017. Optimization of biopharmaceutical downstream processes supported by mechanistic models and artificial neural networks. Biotechnology Progress 33 (3), 696-707.

Püttmann, A., Nicolai, M., Behr, M., von Lieres, E., 2014. Stabilized space-time finite elements for high-definition simulation of packed bed chromatography. Finite Elements in Analysis and Design 86, $1-11$.

Qamar, S., Abbasi, J. N., Javeed, S., Shah, M., Khan, F. U., SeidelMorgenstern, A., 2013. Analytical solutions and moment analysis of chromatographic models for rectangular pulse injections. Journal of Chromatography A 1315, 92-106.

Sellberg, A., Holmqvist, A., Magnusson, F., Andersson, C., Nilsson, B., 2017. Discretized multi-level elution trajectory: A proof-ofconcept demonstration. Journal of Chromatography A 1481, 73 81.

Shipilova, O., Sainio, T., Haario, H., 2008. Particle transport method for simulation of multicomponent chromatography problems. Journal of Chromatography A 1204 (1), 62-71.

von Lieres, E., Andersson, J., 2010. A fast and accurate solver for the general rate model of column liquid chromatography. Computers and Chemical Engineering 34 (8), 1180-1191.

Xu, H., Cantwell, C. D., Monteserin, C., Eskilsson, C., Engsig-karup, A. P., Sherwin, S. J., 2018. Spectral/hp element methods: Recent developments, applications, and perspectives. Journal of Hydrodynamics 30 (1), 1-22.

Zhou, T., Li, Y., Shu, C.-W., 2001. Numerical comparison of weno finite volume and runge-kutta discontinuous galerkin methods. Journal of Scientific Computing 16 (2), 145-171. 